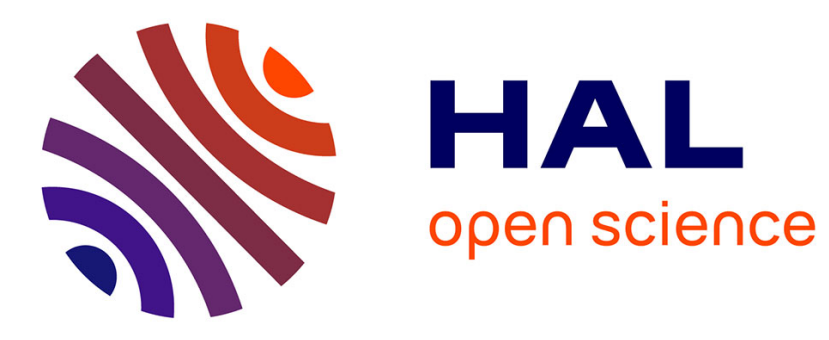

\title{
On numerical simulation of cavitating flows under thermal regime
}

Damien Colombet, Eric Goncalves da Silva, Regiane . Fortes Patella

\section{To cite this version:}

Damien Colombet, Eric Goncalves da Silva, Regiane . Fortes Patella. On numerical simulation of cavitating flows under thermal regime. International Journal of Heat and Mass Transfer, 2017, 105, pp.411-428. 10.1016/j.ijheatmasstransfer.2016.09.070 . hal-01381892

\section{HAL Id: hal-01381892 \\ https://hal.science/hal-01381892}

Submitted on 19 Oct 2016

HAL is a multi-disciplinary open access archive for the deposit and dissemination of scientific research documents, whether they are published or not. The documents may come from teaching and research institutions in France or abroad, or from public or private research centers.
L'archive ouverte pluridisciplinaire HAL, est destinée au dépôt et à la diffusion de documents scientifiques de niveau recherche, publiés ou non, émanant des établissements d'enseignement et de recherche français ou étrangers, des laboratoires publics ou privés. 


\title{
On numerical simulation of cavitating flows under thermal regime
}

\author{
D. Colombet ${ }^{\mathrm{a}, \mathrm{b}, *}$, E. Goncalvès Da Silva ${ }^{\mathrm{b}, * *}$, R. Fortes-Patella ${ }^{\mathrm{b}}$, \\ ${ }^{a}$ CNES, DLA, 52 rue Jacques Hillairet, 75612 Paris Cedex, France \\ ${ }^{b}$ Univ. Grenoble Alpes, LEGI, F-38000 Grenoble, France \\ CNRS, LEGI, F-38000 Grenoble, France
}

\begin{abstract}
In this work, we investigate closure laws for the description of interfacial mass transfer in cavitating flows under thermal regime. In a first part, we show that, if bubble resident time in the low pressure area of the flow is larger than the inertial/thermal regime transition time, bubble expansion are no longer monitored by Rayleigh equation, but by heat transfer in the liquid phase at bubbles surfaces. The modelling of interfacial heat transfer depends thus on a Nusselt number that is a function of the Jakob number and of the bubble thermal Péclet number. This original approach has the advantage to include the kinetic of phase change in the description of cavitating flow and thus to link interfacial heat flux to interfacial mass flux during vapour production. The behaviour of such a model is evaluated for the case of inviscid cavitating flow in expansion tubes for water and refrigerant R114 using a four equations mixture model. Compared with inertial regime (Rayleigh equation), results obtained considering thermal regime seem to predict lower local gas volume fraction maxima as well as lower gradients of velocity and gas volume fraction. It is observed that global vapour production is closely monitored by volumetric interfacial area (bubble size and gas volume fraction) and mainly by the Jakob number variations. It is found that, in contrast with phase change occurring in common boiling flow, Jakob number variation is influenced by phasic temperature difference but also by density ratio variation with pressure and temperature $\left(J a \propto\left(\rho_{L} / \rho_{G}\right) \Delta T\right)$.
\end{abstract}

Keywords: Bubble, Mixture model, Cavitation, Heat/mass transfer, Numerical simulation

\section{Highlights}

- Inception of thermal regime appears very early especially for thermosensitive liquids.

- This enable to consider thermal controlled bubble growth to describe cavitation.

- Vapour production depends closely on local interfacial area and Jakob number.

\footnotetext{
*damien.colombet@univ-grenoble-alpes.fr

** Present address: ENSMA, Poitiers, France, eric.goncalves@ensma.fr

regiane.fortes@univ-grenoble-alpes.fr
} 
- In contrast with common boiling flows, Jakob number is also strongly affected by density ratio variations.

\section{Introduction}

Cavitation is involved in various flow applications such as hydraulic turbines, pumps, rocket turbopump inducers, fuel injectors, marine propellers, underwater bodies, etc. In most of cases, cavitation is an undesirable phenomenon, significantly degrading performance, resulting in lower pressure head of pumps, asymmetric load on turbomachinery blades, vibrations, noise and erosion. In industrial applications, cavitating flows usually take form as a turbulent vapour polydispersed bubbly flow with phase change, bubble break-up and coalescence. In the literature, various gas-liquid mixture or two-fluid models have been developed to investigate isothermal and nonisothermal cavitating flows. According to the assumptions made, those models differ on two main points: equations solved and description of phase change.

Among cavitation models, different approaches can be found to describe phase change due to cavitation: barotropic model [1], short relaxation time model [2], velocity divergence model [3] and model based on inertial [4] or thermal bubble growth [5]. To estimate locally vapour volume fraction, one first approach is to assimilate the gas-liquid mixture to a barotropic fluid. In other words, the density of the gas-liquid mixture is considered to be a function of the local static pressure in the flow. For the simulation of a cavitating flow through a venturi, [1] proposed a sinus barotropic law considering a direct link between the gas volume fraction, phasic densities, local pressure and vapour saturation pressure. For the simulation of cavitating flows in tubopump inducers of spatial rockets, [6] proposed a sinus barotropic law with a vapour saturation pressure calculated from local temperature in the flow. Those robust approaches have provided interesting results for the simulation of hydrofoils [7], venturies [8], turbopump inducers [9, 10, 11], pumpturbines [12] or fuel injectors [13]. Although the simplicity of this modelling approach, this model enable to study complex industrial applications. However, the adaptability of such model for thermosensitive liquids, where temperature gradients are significant, seem to suffer from a lake of physical descriptions of mass and heat transfers induced by phase change at bubbles surfaces.

One second approach is to express explicitly mass and heat transfer terms and to consider that interfacial transfers are instantaneous. In that case, as proposed by [2] or [14], by introducing infinite relaxation parameters (or infinite global transfer coefficients), momentum, mass and heat transfers can be evaluated considering very short equilibrium relaxation times between phasic pressures, velocities, temperatures, Gibbs free energy. This approach, initially devoted for the simulation of diphasic detonation waves [15], is considered to be valid for cavitating flow at very high velocity. As shown recently by [3], very similar results can be achieved considering simply that the mass transfer term is proportional to the mixture velocity divergence. This approach has been recently used for non isothermal cavitation by [16] for the 2D simulations of cavitating flow through a venturi.

To describe cavitation, one last approach is to consider finite rate mass transfer and to express explicitly mass transfer exchange term due to phase change. In the literature, a large number of such cavitation models consider that vapour production in a cavitating flow is only driven by inertial controlled growth of vapour bubbles. For example, considering the equation of [17], a large number of authors $[18,4,19,20]$ have proposed a mass transfer exchange term proportional to the square root of saturated vapour pressure and liquid pressure difference $\left(\sqrt{p^{\text {sat }}-p_{L}}\right)$. Similarly, 
[21] and [22] considered that vapour production depends on phasic pressure difference $\left(p^{\text {sat }}-p_{L}\right)$ and on the convective characteristic time scale of the flow. As shown by [23], for some cases, cavitation models of $[19,20]$ and [22] seem to provide very similar results. Inertial growth models have been also massively employed for the simulation of isothermal cavitating flow through turbopump inducers [24], propellers [25], centrifugal pumps [26, 27], hydrofoils [28, 29, 30] and fuel injectors [31]. For the simulation of cavitating flow in cryogenic fluids, [32] and [33] use the formulation proposed by [21].

Various authors have attempted to take into account the effect of liquid phase thermal gradients in the flow on cavitation. For barotropic approach $[34,6]$ as well as for inertial controlled growth model $[33,35,36]$, it has been done meanly by calculating the saturated vapour pressure as a function of the local temperature $\left(p^{\text {sat }}(T)\right)$ and by estimating the bubble temperature variation using energy balance at the bubble scale. In the same time, a few numerical works evoked that vapour production can be driven by thermal controlled growth of vapour bubbles $[37,38,39,5]$. In [37], authors recall that bubble growth follow two steps. The increase of bubble volume is initially controlled by the liquid inertial (inertial growth) and then is controlled by heat transfer at bubble surface (thermal growth). One of the authors conclusions is that in future works "suitable bubble growth law to model the so-called thermally controlled growth has to be implemented". Conserving short relaxation time for phasic pressures and velocities equilibrium, to take into account finite rate phase change, [38] modified the relaxation model proposed by [2] by including finite rate heat transfer between the dispersed phase and the continuous phase. Later, considering the strong similarity with boiling flow simulations, $[39,5]$ proposed to describe vapour production in cavitating flow assuming only thermal controlled bubble growth. In that approach, the vapour production at bubble surfaces depends on heat flux brought by the liquid and the gas phase.

Despite the large variety of modelling, the numerical simulation of cavitating flow in thermosensitive liquids remains a challenge. Experimentally, for cryogenic and refrigerant fluids, numerous previous works have proved the settling of thermal gradients in the vicinity of cavitating flows [40, 41, 42, 43, 44, 45, 46]. Recent progress in non intrusive temperature measurement in diphasic flow have brought new insights in the field of heat transport in cavitating flow. For cavitating flow trough a micro-diphragme, developing a laser induced two-colour fluorescence method based on themosensitive nano-particles, [47, 48] have managed to access to a 3D mapping of the liquid phase temperature showing the existence of strong temperature gradients near bubble swarms in water at ambient temperature. For hot water, using infra-red measurements, [49] has also confirmed the appearance of hot and cold liquid area in a the cavitating flow trough a macroscopic size venturi. Since the works of [50] and [51] the sensibility of cavitating flow to exhibit liquid phase temperature gradients is often quantified by introducing a parameter named "B factor" (for example $[43,32,33,44,35,6,52]$ ) that corresponds to the ratio between temperature variation in the flow $\Delta T$ and a reference temperature variation $\Delta T^{*}$ as follows

$$
B=\frac{\Delta T}{\Delta T^{*}}, \quad \text { with } \quad \Delta T^{*}=\frac{\rho_{G} L}{\rho_{L} C_{p L}},
$$

where $L$ is the latent heat of vaporization, $\rho_{L}$ and $\rho_{G}$ are respectively densities for the liquid and vapour phases, and $C_{p L}$ represents the specific heat capacity at constant pressure. An increase of B factor is usually associated to intense phase change in cavitating flows. As a mater of fact, 
the description of heat transport and transfers in cavitating flows maybe one of the key to better understand and describe phase change in such complex flows.

The objective of this paper is to investigate phase change model for the simulation of cavitating flow with thermosensitive fluids and especially vapour production. The kinetic of bubble condensation is not considered in the present work. The paper is organized as follows. Section 2 presents and identifies possible closure laws for cavitation modelling considering single bubble growth under phase change for spherical vapour bubbles. Section 3 presents constitutive equations of the proposed mixture (or homogeneous) model. Sections 4 introduces the test case considered to study of the proposed model behaviour : 1D invicid expansion tube problem for cavitation of water and freon R114. Section 5 presents breifly the numerical procedure. Then, section 6 details the results for different flow simulation and try to discuss different points of interest. Section 7 summarizes the main conclusions.

\section{Bubble growth under phase change}

As recalled by $[53,54,55,56,57]$ or [37], bubble growth under phase change follows two consecutive main steps. First, just after nucleation, the bubble growth is controlled by the capacity of the gas phase to struggle against liquid inertia. In other words, the gas phase needs to push the liquid phase to enable the bubble expansion. During this early step, known as "inertial growth", heat transfers at bubble surface are considered to be non limiting. Then, at a given time $\tau$, bubble growth is controlled by the capacity of the fluid (gas and liquid) to bring enough heat at the bubble surface to enable phase change. During this second step, known as "thermal growth", heat transfers

control the bubble expansion, and mechanical energy necessary for the bubble growth is considered to be non limiting. 


\subsection{Inertial growth}

As shown by [17] and [58], solving the momentum conservation equation with central symmetry for a spherical bubble, neglecting interfacial viscous stress, Laplace pressure jump and dissolved gas in the liquid phase, for an incompressible fluid, during inertial growth the bubble radius time evolution verifies the following differential equation

$$
\rho_{L}\left[R \frac{d^{2} R}{d t^{2}}+\frac{3}{2}\left(\frac{d R}{d t}\right)^{2}\right]=p_{L(r=R)}-p_{L(r=\infty)},
$$

where $p_{L(r=\infty)}$ is the pressure in the far field of the liquid phase and $p_{L(r=R)}$ is the pressure in the liquid phase at the bubble surface. Eq. 2 is known as Rayleigh-Plesset equation. Considering that $p_{L(r=\infty)}=p_{L}$ corresponds to bulk liquid phase pressure and that $p_{L(r=R)}=p^{\text {sat }}\left(T_{L}\right)$ corresponds to the pressure at saturation given for the liquid bulk temperature $T_{L}$, with a small initial bubble radius $(R(t=0)<<R)$, integration of equation 2 shows that the bubble radius time derivative is

$$
\left(\frac{d R}{d t}\right)_{\text {inertial }}=\sqrt{\frac{2}{3} \frac{\Delta p}{\rho_{L}}} \quad \text { with } \quad \Delta p=p^{\text {sat }}\left(T_{L}\right)-p_{L}
$$

Note that Laplace pressure jump can only be neglected for large enough bubble when $(2 s / R) /\left[p_{L}-\right.$

$\left.p^{\text {sat }}\left(T_{L}\right)\right]<<1$, where $s$ is the surface tension of the fluid. In the same way, the viscous normal stress can be neglected for moderate pressure decrease and low viscosity fluids when $\left(10 \mu^{2}\right) /\left(R^{2} \rho_{L}\left[p_{L}-\right.\right.$ $\left.\left.p^{\text {sat }}\left(T_{L}\right)\right]\right)<<1$. According to Eq. 3, during inertial growth, for a given liquid density and pressure difference $\Delta p$, the bubble radius increases as $R \propto t$. Consequently, equation 3 corresponds to interfacial velocity during inertial growth just after bubble nucleation.

\subsection{Thermal growth for a fixed bubble}

At the gas-liquid interface scale, the respect of energy conservation shows that heat required for phase change corresponds to the difference between interfacial heat fluxes as follows [59]

$$
L J_{L o c}=\lambda_{L} \nabla T_{L} \cdot \vec{n}-\lambda_{G} \nabla T_{G} \cdot \vec{n},
$$

where $J_{L o c}$ is the local mass flow rate per surface unit, $L=h_{G 0}-h_{L 0}$ is the latent heat of vaporization, $\lambda_{k}$ stands for the thermal conductivity of phase $k$ and $\vec{n}$ is the vector normal to the interface. Therefore, at the bubble surface, heat flux coming from each phase depends on thermal conductivities and temperature normal gradients. For common cavitating fluids, one can show that thermal conductivity of the liquid phase $\lambda_{L}$ is several times larger than the one of the gas phase $\lambda_{G}$ (see Tab. 1). Thus, most of the part of heat necessary for phase change comes from the liquid phase and gas phase heat flux can be neglected so that $J_{L o c} \approx \lambda_{L} \nabla T_{L} \cdot \vec{n} / L$ (Eq. 4). Due to the low thickness of the gas-liquid interface, at the bubble surface, thermodynamic equilibrium is verified. The interfacial temperature corresponds therefore to the saturated vapour temperature based on the local liquid pressure so that $T^{I}=T^{\text {sat }}\left(p_{L}\right)$. In addition, for common cavitating fluids, gas phase thermal diffusivity $D_{G}^{t h}=\lambda_{G} /\left(\rho_{G} C p_{G}\right)$ stays much larger than the liquid phase thermal diffusivity $D_{L}^{t h}=\lambda_{L} /\left(\rho_{L} C p_{L}\right)$ (see Tab. 1). It is thus possible to consider that the gas phase temperature inside bubbles corresponds to the one settled at the gas-liquid interface so that $T_{G(r \leq R)}=T_{G(r=R)}=T_{L(r=R)}=T^{I}=T^{\text {sat }}\left(p_{L}\right)$. 
Under those two assumptions, mass flux at the bubble surface is meanly controlled by heat flux provided by the liquid phase (i.e. heat transfer around the bubble). At the bubble scale, the enthalpy balance can thus be written as follows

$$
\frac{d\left(V_{b} L \rho_{G}\right)}{d t}=S_{b} H_{L}\left(T_{L(r=\infty)}-T_{L(r=R)}\right)
$$

with the bubble volume $V_{b}=4 \pi R^{3} / 3$ and surface $S_{b}=4 \pi R^{2}, \rho_{G}$ the gas density, $H_{L}$ the heat transfer coefficient in the liquid phase (in $W m^{-2} K^{-1}$ ), $T_{L(r=\infty)}=T_{L}$ the liquid phase temperature far away from the bubble surface. Introducing the Nusselt number $N_{u L}$ that quantified normalized heat flux around the bubble, the development of this equation with $T_{L(r=R)}=T^{s a t}\left(p_{L}\right)$ and $\Delta T=T_{L}-T^{s a t}\left(p_{L}\right)$ gives the following interfacial velocity

$$
\frac{d R}{d t}=\left(\frac{N_{u L} \lambda_{L}}{2 R}\right) \frac{\Delta T}{\rho_{G} L} \quad \text { with } \quad N_{u L}=\frac{H_{L}(2 R)}{\lambda_{L}}
$$

Assuming a fixed bubble (no buoyancy effect), taking into account only the bubble growth, [60] shows analytically that the time derivative of the bubble radius depends on the Jakob number $J a$ as follows

$$
\left(\frac{d R}{d t}\right)_{\text {thermal }}=\sqrt{\frac{4 J a^{2} f(J a) D_{L}^{t h}}{\pi t}} \quad \text { with } \quad J a=\frac{\rho_{L} C p_{L} \Delta T}{\rho_{G} L},
$$

with (see [57])

$$
f(J a)=\frac{\pi F}{8 J a^{2}}
$$

where $F$ is an implicit value given by solving the folowing equation

$$
J a=F \exp \left(\frac{3}{2} F\right) \int_{1}^{\infty} \frac{1}{x^{2}} \exp \left(-\frac{F}{x}-\frac{F}{2} x^{2}\right) d x
$$

The Jakob number compares sensible heat available in a volume of liquid corresponding to the bubble volume $\left(V_{b} \rho_{L} C p_{L}\left(T_{L}-T^{s a t}\left(p_{L}\right)\right)\right)$ to latent heat necessary to form the bubble under phase change $\left(V_{b} \rho_{G} L\right)$. Thus, an increase of Jakob number corresponds to an increase of heat and vapour mass transfer rates. As already reported by [61] and [62], it is very interesting to note that Jakob number usually employed to study phase change in boiling flows corresponds in fact to the definition of the B-factor (Eq. 1) used for the study of cavitating flows with thermal effects. And, the temperature variation $\Delta T^{*}$ used to characterise the importance of thermal effects in cavitating flows is thus the temperature variation corresponding to a Jakob number equals to unity $\mathrm{Ja}=1$. This observation shows us how boiling and cavitating flows are close since both kind of flow follows the physic of phase change.

Thus, during thermal growth, for given liquid phase properties and a given Jakob number, the bubble radius increases as $R \propto t^{1 / 2}$. Consequently, equation 7 corresponds to interfacial velocity during thermal growth, without slip induced by buoyancy between the gas and the liquid phase. 
Assuming a small initial radius $(R(t=0)<<R)$, the corresponding Nusselt number based on Scriven's result is (Eq. 7)

$$
N_{u L 0}=\frac{2 \rho_{G} L}{\lambda_{L} \Delta T} R \frac{d R}{d t}=\frac{16}{\pi} J a f(J a)
$$

We have reported in Fig. 1 this Nusselt number as a function of the Jakob number (-). As shown in this figure, for low $J a$, the Nusselt number tends logically to the pure diffusion solution $N_{u L 0}(J a \rightarrow 0)=2$. Then, when the Jakob number increases, typically for $J a>10$, the Nusselt number tends gradually to the solution identified earlier by [63]: $N_{u L 0}(\mathrm{Ja} \rightarrow \infty)=12 \mathrm{Ja} / \pi$. As depicted in Fig. 1, Scriven's solution is found to be in agreement with direct numerical simulation performed by [57] (०) describing the thermal growth for a fixed bubble. However, the analytical solution found by [60] (Eq. 8-9) has the drawback to be implicit. An approximation of Scriven's result has been given by [64] as $f(J a)=\pi /(8 J a)+(1 / 16)\left[6 \pi^{2} / J a^{2}\right]^{1 / 3}+3 / 4$.

The corresponding Nusselt number is (Eq. 10)

$$
N_{u L 0}=2+\left(\frac{6 J a}{\pi}\right)^{1 / 3}+\frac{12}{\pi} J a
$$

As shown in Fig. 1, this approximation is in very good agreement with the analytical solution describing the two asymptotic solutions at low and high $J a$ and the transition between them $(-\times)$.

\subsection{Thermal growth for a bubble with slip}

The above description of thermal growth kinetic remains valid as long as the interfacial velocity $d R / d t$ stays much larger than the relative velocity between the bubbles and the liquid phase $v_{r}=$ $v_{G}-v_{L}$ [57]. For that case, the heat transport at the bubble surface occurs essentially radially to the bubble centre. Then, at a given time $\tau^{\prime}$ during bubble thermal growth, the relative velocity of bubbles $\left(v_{r}\right)$ can generate additional heat transport tangentially to the bubble surface. As shown by [57] and as reported in Fig. 2, when advection due to bubble slip begins to affect heat transfer, the Nusselt number increases gradually with instantaneous Péclet number $\operatorname{Pe}(t)=\operatorname{Re}(t) \operatorname{Pr}$ from the Scriven's solution to the analytical solution identified by [65] for $(d R / d t) / v_{r}<<1$ and $\operatorname{Re}(t)=$ $2 v_{r}(t) R(t) / \nu_{L}>>1$

$$
N_{u L}=\frac{2}{\sqrt{\pi}} P e(t)^{1 / 2}
$$

where $\operatorname{Pr}=\nu_{L} / D_{L}^{t h}$ is the liquid phase Prandlt number that compares momentum and heat diffusions. This behaviour was recently confirmed by [66] using Level Set/Ghost Fluid numerical simulations for a spherical bubble. For this regime, bubble volume time variation is given by introducing Eq. 12 in Eq. 6

$$
\left(\frac{d R}{d t}\right)_{\text {thermal with slip }}=\frac{J a D_{L}^{t h}}{R \sqrt{\pi}} P e(t)^{1 / 2}
$$

Considering Levich's drag for a spherical bubble [67], we get $v_{r} \propto R^{2}$, and one can show using Eq. 13 that, for thermal growth enhanced by the liquid advection around bubbles $\left((d R / d t) / v_{r}<<\right.$ 
1), bubble radius increases as $R \propto t^{3 / 2}$. Recently, to describe the smooth transition found by [57] between Scriven's and Ruckenstein's results, [68] proposed the following empirical equation

$$
N_{u L}=N_{u L 0}\left(1+\left(\frac{P e}{P e_{c}}\right)^{n / 2}\right)^{1 / n} \quad \text { with } \quad P e_{c}=\frac{\pi N_{u L 0}^{2}}{4},
$$

with $n \approx 2.65$. The critical Péclet number $P e_{c}$ corresponds to the Péclet number for which we get the equality between Labuntsov's Nusselt approximation and Ruckenstein's solution. This relation is found to be in agreement with direct numerical simulations of [57] for thermal growth with a slip velocity. Consequently, under phase change, the bubble growth can be thought in three steps : inertial growth $(t<\tau, R \propto t)$, thermal growth without slip effect $\left(\tau<t<\tau^{\prime}, R \propto t^{1 / 2}\right)$, thermal growth enhanced by bubble slip $\left(t>\tau^{\prime}, R \propto t^{3 / 2}\right)$. In order to describe cavitating flow in thermosensitive fluids, the next question of importance is to know how long is the inertial growth. This is the objective of the next section.

\subsection{Inception of thermal growth}

Assuming that the transition between inertial and thermal growth regimes is very short, it is possible to estimate the time $\tau$ corresponding to the inception of thermal growth considering the equality between interfacial velocities $(d R / d t)_{\text {inertial }}=(d R / d t)_{\text {thermal }}$ given respectively by Eq. 3 and 7 as

$$
\tau=\frac{6}{\pi} \frac{\rho_{L} f(J a) J a^{2} D_{L}^{t h}}{\Delta p}
$$

where the pressure difference can be linked to a temperature difference as follows

$$
\Delta p=p^{s a t}\left(T_{L}\right)-p_{L}=\frac{d p^{s a t}}{d T}\left(T_{L}-T^{s a t}\left(P_{L}\right)\right)=\frac{d p^{s a t}}{d T} \Delta T
$$

with $d p^{s a t} / d T$ corresponding to the slope of the linearised saturation curve ( $p^{\text {sat }}$ vs $T$ ). It is very interesting to note that the time $\tau$ for which heat transfer prevails on inertial effects appears to be independent to the bubble radius. In Fig. 3, we have estimated this time as a function of the Jakob number for different common cavitating fluids. For this calculation, fluid properties $\left(\rho_{L}, \rho_{G}, C_{p L}\right.$, $\left.\lambda_{L}, L, d p^{s a t} / d T\right)$ are considered for $T=T_{0}$ and at saturation. First, we can observe that curves get the same shape and exhibit a vertical shift between them. For $J a<0.1$, it is found that for cryogenic liquids (nitrogen, oxygen or hydrogen), refrigerant freon R114 and hot water the thermal growth appears for a time larger than $t>1 \mu \mathrm{s}$ during the bubble growth. At low Jakob number one can show that $\tau$ tends to the constant value $\tau(J a<<1)=(3 / 4)\left(\rho_{L}^{2} C_{p L} D_{L}^{t h}\right) /\left(\left(d p^{s a t} / d T\right) \rho_{G} L\right)$. For $1<\leq J a \leq 100$, it is shown that for hot water $(T=355 \mathrm{~K})$ and water at ambient temperature $(T=293 K)$ thermal growth appears later (at $1 \mu s \leq t \leq 0.01 s$ ) while for other thermosensitive fluids $\tau$ stays lower than $1 \mu \mathrm{s}$. For larger Jakob number, $\tau$ begins to be proportional to $J a$ so that $\tau(J a>>1)=[9 /(2 \pi)]\left(\rho_{L}^{2} C_{p L} D_{L}^{t h}\right)\left(\left(d p^{s a t} / d T\right) \rho_{G} L\right) J a \propto J a$. Thus for the range $0.001<$ $J a<100$, except for water at ambient temperature, the transition from inertial growth to thermal regime appears for $\tau<0.1 \mathrm{~ms}$. This time of transition $\tau$ needs to be compared to the resident 
time corresponding to the transport of bubbles $\tau_{\text {res }}=l / u$, whith $l$ is the length of bubble path in the low pressure area where $p<p^{\text {sat }}$ in cavitating flow and $u$ the mean flow velocity along that path. For the case of $\tau / \tau_{\text {res }}<<1$, thermal controlled bubble growth should be considered to describe vapour production instead of inertial growth. It is clear that $\tau_{\text {res }}$ depends on the nature of the flow. As a consequence, in the following, thermal controlled growth closure law is investigated for cavitating flows caracterised by $\tau / \tau_{\text {res }}<<1$. As shown by Fig. 3, this condition is likely to be fulfilled for cavitation in hot water and cryogenic fluids.

\section{Modelling of non-isothermal cavitating flow}

In this section we present a mixture model based on the simplification of the six equations twofluid model introduced by [59]. The reader can refer to [69] in order to have a complete description of the parent two-fluid model. Then, considering different hypothesis, we introduce the closure of the mixture model.

\subsection{The Euler mixture model}

In this work, for the sake of simplicity in order to test the different existing closure laws for mass transfer, we neglect viscous and heat diffusion effects at the scale of the bubbly flow. In addition, as usual with hydrodynamic cavitation, we are interested to liquid flow at large velocity compared to bubble relative velocity $\left(v_{r} / v_{L}=\left(v_{G}-v_{L}\right) / v_{L}<<1\right)$ induced by buoyancy effect. As a result, in such flow we can assume the equality between phasic velocities by introducing the mixture velocity $v_{m}=v_{L}=v_{G}$. In the present mixture model, by introducing the mixture density $\rho_{m}=\alpha_{G} \rho_{G}+\left(1-\alpha_{G}\right) \rho_{L}$, we will consider the mass conservation equation of the gas-liquid mixture

$$
\frac{\partial \rho_{m}}{\partial t}+\nabla \cdot\left(\rho_{m} v_{m}\right)=0
$$

This equation results from the sum of phasic mass conservation equations, with the equality of phasic mass transfer terms $\Gamma_{G}=-\Gamma_{L}$. Since we consider large velocity flows, gravity effect in momentum conservation equations can also be neglected. In addition, viscous and turbulent momentum or heat phasic diffusive fluxes are neglected compared to advective fluxes. In other words, at the scale of the cavitating flow, we consider a perfect fluid and Euler conservation equations. By summing phasic momentum conservation equations, with the equality of phasic momentum transfer terms, we consider the momentum conservation of the gas-liquid mixture,

$$
\frac{\partial\left(\rho_{m} v_{m}\right)}{\partial t}+\nabla \cdot\left(\rho_{m} v_{m} v_{m}\right)=-\nabla p
$$

In the same way, we will consider the conservation of the mixture total energy $\rho_{m} E_{m}=\alpha_{G} \rho_{G} E_{G}+$ $\left(1-\alpha_{G}\right) \rho_{L} E_{L}$

$$
\frac{\partial\left(\rho_{m} E_{m}\right)}{\partial t}+\nabla \cdot\left(\rho_{m} v_{m} E_{m}\right)=\nabla \cdot\left(p I . v_{m}\right)
$$

with $E_{k}=h_{k}\left(T_{k}\right)-p / \rho_{k}+v_{k}^{2} / 2$ the total energy of phase $k$ that corresponds to the sum of internal energy $\left(e_{k}=h_{k}\left(T_{k}\right)-p / \rho_{k}\right)$ with kinetic energy $\left(v_{k}^{2} / 2\right)$. This equation results from the sum 
of phasic energy conservation equations, with the equality of phasic energy transfer terms. For an incompressible fluid, solving mass conservation equation 17 gives a direct access to the gas volume fraction. But in the present approach, gas and liquid phase are considered to be compressible. Consequenly, it is necessary to add one equation to access to the gas volume fraction $\alpha_{G}$. In the literature, for numerical simulations of compressible diphasic flow, numerous authors $[70,71,16]$ have used the following gas volume fraction transport equations proposed by [72]

$$
\begin{aligned}
& \frac{\partial \alpha_{G}}{\partial t}+v_{m} \nabla \cdot\left(\alpha_{G}\right)=K \nabla \cdot\left(v_{m}\right)+\frac{1}{\rho_{I}} \Gamma_{G}, \\
& \text { with } K=\frac{\alpha_{G} \alpha_{L}}{\alpha_{G}+c_{G}^{2} \rho_{G} /\left(c_{L}^{2} \rho_{L}-c_{G}^{2} \rho_{G}\right)} \quad \rho_{I}=\frac{\alpha_{G} c_{L}^{2}+\alpha_{L} c_{G}^{2}}{\alpha_{G} c_{L}^{2} \rho_{L}+\alpha_{L} c_{G}^{2} \rho_{G}},
\end{aligned}
$$

where $c_{k}=\sqrt{\left(\gamma_{k}-1\right) C_{p k} T_{k}}$ is the sound speed based on mass heat capacity at constant pressure $C_{p k}$ and Laplace coefficient $\gamma_{k}=C_{p k} / C_{v k}$ in each phase $k$. The above transport equation is established from phasic mass conservation equations assuming the equality between total eulerian derivative of pressure in each phase. One can note that for $c_{L}^{2}>>c_{G}^{2}$ and $\rho_{L} c_{L}^{2}>>\rho_{G} c_{G}^{2}$, we have $K \approx \alpha_{L}$ and $1 / \rho_{I} \approx 1 / \rho_{L}$ so that Kapila transport equation (20) tends to mass conservation equation for the case of an incompressible liquid phase. The unknown variables of this model are

$\alpha_{G}, \rho_{G}, \rho_{L}, T_{G}, T_{L}, p, v_{m}$. To close this model it is necessary to describe mass transfer due to phase change $\Gamma_{G}$ and to choose two equations of states for the calculation of densities. This modelling approach requires to solve 4 equations.

\subsection{Equation of state}

In this work, the following stiffened gas equation of state is used for both phases ([73])

$$
p\left(\rho_{k}, e_{k}\right)=\left(\gamma_{k}-1\right) \rho_{k}\left(e_{k}-\pi_{k}\right)-\gamma_{k} p_{k}^{\infty},
$$

with $e_{k}$ internal energy of phase $k, \pi_{k}=h_{k 0}-C_{p k} T_{0}$ an internal energy of reference based on the enthalpy of formation $h_{k 0}$ at the initial temperature $T_{k}=T_{0}, p_{k}^{\infty}$ a pressure of reference. Parameters for using the stiffened gas equation of state are given in Tab. 2. Note that, for the gas phase with $p_{G}^{\infty}=0$, the use of stiffened gas equation of state is equivalent to the use of perfect gas equation of state.

\subsection{Calculation of gas phase temperature}

As explained in section 2.2, the gas phase temperature is considered to be homogeneous in the bubble $\left(T_{G}=T^{I}\right)$ and it corresponds to saturation temperature given by the local pressure using here Antoine's equation as follows [74]

$$
T_{G}=T^{I}=T^{s a t}(p)=B[A-\log (p)]^{-1}-C,
$$

with $p$ expressed in Torr, where constants are given in Tab. 3. This approach for the estimation of gas phase temperature is only valid up to the triple point that corresponds to pressure and temperature given in Tab. $4\left(T^{T}\right.$ and $\left.p^{T}\right)$. Below this limit, phase change, such as solidification of liquid and gas, may appear. 


\subsection{Mass transfer term for thermal growth}

Considering interfacial balances of mass and energy, one can show the link between interfacial phasic mass transfer terms $\left(\Gamma_{G}, \Gamma_{L}\right)$ and interfacial phasic heat transfer flux $\left(q_{L}^{\prime \prime}, q_{G}^{\prime \prime}\right)[5]$

$$
\Gamma_{G}=-\Gamma_{L}=-\frac{\left(q_{L}^{\prime \prime}+q_{G}^{\prime \prime}\right)}{\left(E_{G i}-E_{L i}\right)},
$$

with $\left(E_{G i}-E_{L i}\right)$ the difference of total energy at the bubble surface that is usually approximated as the enthalpy difference at the initial temperature of the flow $\left(E_{G i}-E_{L i}\right) \approx h_{G 0}-h_{L 0}=L$. As reported before in section 2.2 , since conductivity in the liquid phase is much larger than in the gas phase, heat flux supplied by the gas phase at the bubble surface $q_{G}^{\prime \prime}$ can be neglected compared to heat flux supplied by the liquid phase $q_{L}^{\prime \prime}$ so that

$$
\Gamma_{G} \approx-\frac{q_{L}^{\prime \prime}}{L}=-\frac{a_{I} H_{L}\left(T^{I}-T_{L}\right)}{L} \quad \text { with } \quad H_{L}=\frac{\lambda_{L} N_{u L}}{d_{b}}
$$

In addition, as written in the equation above, heat flux in the liquid phase at the bubble surface is a function of the difference of temperature between the gas and the liquid $\left(T^{I}-T_{L}\right)$, interfacial area per volume unit $a_{I}$ and heat transfer coefficient $H_{L}$ in the liquid phase that is monitored by the bubble Nusselt number. The Nusselt number can be estimated with Eq. 14 where relative velocity between the bubbles and the liquid phase $v_{r}$ is estimated from physical properties at saturation with the correlation of [75] considering the balance between buoyancy and drag forces applied on bubbles.

Moreover, in this work, we consider flows where cavitation takes place as a dispersion of bubbles that can be characterised by a mean Sauter diameter an average reference bubble diameter $d_{b}$ that results from bubbles growth, collapse, break-up and coalescence phenomena. This reference diameter is consider to be representative of the bubble dispersion. The mean Sauter diameter that corresponds to the ratio between the third and second moment of the bubble size distribution is considered to be representative of the interfacial area of the bubble dispersion. The interfacial exchange area per volume unit is thus calculated as $a_{I}=6 \alpha_{G} / d_{b}$. Finally, for comparison with this thermal regime closure law, we will also consider results obtained with an inertial regime closure law, with velocity divergence model or with infinite relaxation parameter model. For the sake of simplicity, in the following, the mean Sauter diameter $d_{b}$ will be called simply bubble diameter.

\subsection{Mass transfer term for inertial growth}

Even if inertial growth is only valid at the early age of bubbles, from Eq. 3, one can define the corresponding mass transfer term as follows

$$
\Gamma_{G}=\rho_{G} \frac{d \alpha_{G}}{d t}=a_{I} \frac{d R}{d t}=\rho_{G} a_{I} \sqrt{\frac{2}{3} \frac{\Delta p}{\rho_{L}}} \quad \text { with } \quad \Delta p=p^{s a t}\left(T_{0}\right)-p_{L}
$$

where $\rho_{L}, \rho_{G}$ and $p^{\text {sat }}\left(T_{0}\right)$ are taken in this work as constant and equal to saturation values at $T=T_{0}$. This closure for mass transfer term is very close to the one proposed by [4] or [19] that, 
instead of fixing a bubble diameter, preferred to impose the nuclei concentration per unit volume of pure liquid. This source term will be larger than the one based on thermal controlled growth (Eq. 23). It is numerically very stiff generating easily oscillation of the solution. For this reason, it was only possible to compare it to cases with low stretching velocity. Note that in this work only growth of bubble under cavitation is studied. Thus, only positive source term is considered and $\Gamma_{G}$ is set to zero elsewhere.

\subsection{Mass transfer term proportional to velocity divergence}

Recently, [3, 16] has proposed a mass transfer source term for vapour production and condensation by assuming $\Gamma_{G}$ to be proportional to the divergence of the velocity as follows

$$
\Gamma_{G}=\left(1-\frac{c_{E O S}^{2}}{c_{W}^{2}}\right) \frac{\rho_{L} \rho_{G}}{\rho_{L}-\rho_{G}} \nabla \cdot v_{m},
$$

where $c_{E O S}$ is the speed of sound given by a mixture equation of state and $c_{W}$ is the speed of sound proposed by [76] and [77] for a bubbly flow

$$
c_{W}^{2}=\frac{1}{\rho_{m}} \frac{\rho_{L} \rho_{G} c_{L}^{2} c_{G}^{2}}{\alpha_{G} \rho_{L} c_{L}^{2}+\alpha_{L} \rho_{G} c_{G}^{2}},
$$

\subsection{Mass transfer term based on an infinite relaxation parameter}

For cavitation generated at very large flow velocity, considering the compressible two-fluid model introduced by [15], [71] or [14] proposed to model heat transfer by introducing a relaxation parameter $\theta$ for phasic temperature. As a result, heat transfer associated to phase change is expressed as $\theta\left(T_{L}-T^{I}\right)$ where $\theta$ in $W m^{-3} K^{-1}$ is assumed to be infinite. In other words, this kind of approach gives the asymptotic result for an instantaneous mass and heat transfers between phases.

\section{Presentation of the expansion tube test case}

To investigate cavitation in thermal regime we need to choose a test case that verify the condition $\tau / \tau_{\text {res }}<<1$. Among test cases existing in the literature, the expansion tube test case depicted in Fig. 4 is particularly interesting since velocity of the flow in the centre of the tube, where cavitation takes place, is cancelled so that resident time of bubbles $\tau_{\text {res }}$ is very large compared to the time required for the inception of thermal regime $\tau$. In addition, this case has been already studied previously for hot water by [73] and [14] with respectively 5 and 7 equations models, where momentum and mass transfer terms are described using infinite relaxation parameter. Recently, this test case was also calculated by $[3,16]$ with a 4 equations model using a mass transfer source term proportional to mixture velocity divergence for hot water and refrigerant freon R114. This test case consists in a one meter long tube filled with water or R114 with physical properties and initial conditions given in Tab. 4. To stretch the liquid initially at rest, a velocity is imposed at the right and left hand side extremities. The initial velocity discontinuity is set at $x=0.5 \mathrm{~m}$ so that $v_{m}(x \leq 0.5 \mathrm{~m})=-u$ and $v_{m}(x<0.5 \mathrm{~m})=u$. To be able to initiate phase change $\left(a_{I}>0\right)$, a weak volume fraction of vapour $\alpha_{G}=0.01$ is initially added to the liquid. The choice of R114 as a second test fluid is motivated because it is expected to be more thermosensitive than hot water (larger $\Delta T^{*}$, Tab. 4). 
As reported by Eq. 24 or 25 , mass transfer term $\Gamma_{G}$ closely depends on interfacial area and thus on the reference bubble diameter $d_{b}$. Consequently, in the following, the bubble diameter will be varied to appreciate the effect of this parameter on the intensity of phase change.

\section{Numerical procedure}

The conservation laws governing this mixture model can be written in the form

$$
\frac{\partial w}{\partial t}+\nabla \cdot F(w)=S
$$

where $w$ is the vector of variables, $F$ the convective flux and $S$ the source term (only for the gas volume fraction equation). The gas volume fraction equation (Eq. 20) is written in its divergence form

$$
\frac{\partial \alpha_{G}}{\partial t}+\nabla \cdot\left(\alpha_{G} v_{m}\right)=\left(K+\alpha_{G}\right) \nabla \cdot\left(v_{m}\right)+\frac{1}{\rho_{I}} \Gamma_{G},
$$

A regular mesh is considered, whose size $\Delta x$ is such that: $\Delta x=x_{i+1 / 2}-x_{i-1 / 2}$. The time step complies with CFL condition in order to guarantee the stability requirement. $\Delta t$ denotes as usually the time step, where $\Delta t=t^{n+1}-t^{n}$. Let $w_{i}^{n}$ be the approximate value of $(1 / \Delta x) \int_{x_{i-1 / 2}}^{x_{i+1 / 2}} w\left(x, t^{n}\right) d x$. A discrete form of Eq. 28 can be written in $1 \mathrm{D}$ as

$$
\Delta x \frac{\left(w_{i}^{n+1}-w_{i}^{n}\right)}{\Delta t}+F_{i+1 / 2}^{n}-F_{i-1 / 2}^{n}=S_{i}^{n} \Delta x
$$

where $F_{i+1 / 2}^{n}$ is the numerical flux through the cell interface $x_{i+1 / 2}$ at time $t^{n}$. Various formulations of numerical flux have been proposed to solve multiphase compressible flows. In the present study, we consider the formulation based on the Rusanov scheme [78] with a mesh composed of 5000 cells. Various tests on grid spacing influence have been performed with different meshes. As reported in Tab. 5, a grid composed of more than 4000 cells is sufficiently fine to tackle grid dependence effect on final results.

\subsection{Treatment of the source term}

The numerical simulations of the initial-boundary value problems are accomplished using the splitting approach. One starts in solving the source-free homogeneous part of the whole system

$$
\frac{\partial w}{\partial t}+\nabla \cdot F(w)=0
$$

This is followed by solving the system of ordinary differential equations describing the mass transfer between phases to obtain the complete solution

$$
\frac{d w}{d t}=S(w)
$$

\subsection{Initial and boundary conditions}

Initially, we impose the gas volume fraction $\left(\alpha_{G}=0.01\right)$ but also the densities of pure phases and the velocity profiles. At the position $x=0$, the fluid velocity is imposed and we cancel the gradients of pressure, gas volume fraction and mixture density. At the position $x=1 \mathrm{~m}$, the pressure is imposed and we cancel the gradients of mixture velocity, gas volume fraction and mixture density. 


\section{Results and discussions}

The first subsection presents results obtained from the model for hot water in the expansion tube by considering no mass transfer between the gas and the liquid phase. Then, the second subsection presents and analyses results obtained for cavitation of hot water and refrigerant R114. A comparison of results obtained with different closure laws for mass transfer is proposed. The third subsection analyses the mechanism of phase change in the cavitating flow. In the fourth and fifth subsections, the influence of the heat advection at bubbles surface and the influence of fluid properties variation in the calculation of Jakob number are discussed. Finally, in the last subsection, the behaviour of the model for simulations at larger stretching velocity is achieved.

\subsection{Rarefaction test case without mass transfer}

In order to appreciate compressibility effects on cavitating flow, the first simulations are performed by cancelling the mass transfer term $\left(\Gamma_{G}=0\right)$. Velocity, pressure, gas volume fraction and gas density profiles obtained for hot water at $T=355 \mathrm{~K}$ with a stretching velocity of $|u|=2 \mathrm{~ms}^{-1}$ are displayed in Fig. 5 for the different times $t=10^{-4}$, 0.8, 1.6, $3.2 \mathrm{~ms}$ and for initial conditions presented in Tab. 4. As reported in this figure, while the velocity profile is developing, the pressure in the centre of the tube decreases strongly. But since a gas phase is present, the pressure cannot become negative. As already noticed by [73] or [14], this decrease of pressure induced an important decrease of gas phase density $\rho_{G}$ that is traduced as a peak of gas volume fraction up to $\alpha_{G} \approx 10 \%$ in the centre of the tube. This test case without mass transfer shows us how compressibility effects (i.e. gas phase expansion) take part in the variation of gas volume fraction in real cavitating flows. As a result, the advantage of solving compressible formulation of equations is that it enables to take into account both the phase change and the gas phase density variation with pressure and temperature. Finally, we can notice that our results for $t=3.2 \mathrm{~ms}$ are found to be in perfect agreement with the previous work of [14] (o) using a 7 equations two-fluid model.

\subsection{Simulation at moderate stretching velocity}

In this section we consider the same case than previously: cavitation of hot water at $T=355 \mathrm{~K}$ with a stretching velocity of $|u|=2 \mathrm{~ms}^{-1}$, but now with mass transfer $\left(\Gamma_{G} \geq 0\right)$. From Eq. 15, we get $\tau \approx 10^{-7}-10^{-4} s$ (Fig. 3) and $\tau_{\text {res }}=l / u \approx 0.5 s$ so that the condition $\tau / \tau_{\text {res }}<<1$ is verified for that case and a thermal growth closure law should be employed.

Velocity, pressure, gas volume fraction and liquid phase temperature profiles obtained for this case are displayed in Fig. 6 for the the time $t=3.2 \mathrm{~ms}$. Even if in the present flow conditions, bubble growth is in a thermal regime, it is interesting to compare results given by an inertial bubble growth since in the literature phase change kinetics based on phasic pressure difference are the most used modeling approach for cavitation. Thus, we will first consider results obtained by using inertial bubble growth as a closure law for mass transfer (Eq. 25, red continuous line), with a reference diameter of $d_{b}=0.5 \mathrm{~mm}$. Compared to the previous test case (Fig. 5), because of mass transfer and vapour production, the gas volume fraction reaches a value near $\alpha_{G}=70 \%$ instead of $10 \%$ in the centre of the tube. Moreover, in the centre of the tube the pressure tends to the initial vapour saturated pressure $\left(p_{s a t 0}=0.51 \mathrm{bar}\right)$. The velocity profile is sharper with mass transfer than without. And for such case, a liquid phase temperature decrease of less than one Kelvin is induced by phase change. For comparison, results found by [14] (०) with the infinite relaxation parameter model and by [3] $(\square)$ with the velocity divergence model are also reported in this figure. 
It is very interesting to notice that globally the solution obtained with the inertial model (Eq. 25) is very close to the one obtained previously by [14] and [3] that correspond to a pseudo instantaneous mass transfer. This is due to the fact that mass transfer rate predicted by inertial growth are so important that it is equivalent to considering an infinite rates of heat and mass transfers.

Secondly, we can now consider results obtained by using thermal bubble growth as a closure law for mass transfer (Eq. 24 and 14) with three different reference bubble diameters $d_{b}=0.5-$ $3-10 \mathrm{~mm}$. One can notice that modifying the reference bubble diameter affect the whole final solution. Increasing $d_{b}$ results in smoothing the velocity profile. In addition, in the centre of the tube the pressure tends to value lower or near the vapour saturated pressure and the production of vapour results in a gas volume fraction peak of $\alpha_{G} \approx 30-70 \%$ depending the reference bubble diameter chosen. Fig. 7 presents the gas volume fraction obtained in the centre of the tube at $x=0.5 \mathrm{~m}$ and $x=0.45 \mathrm{~m}$ for $t=3.2 \mathrm{~ms}$ as a function of the chosen bubble reference diameters (continuous lines). As reported in Fig. 7a, we can notice that the larger is the reference bubble diameter, the smaller is the maximal value of gas volume fraction in the centre of the tube. In the opposite, Fig. 7b shows that the increase of the reference bubble diameter produces a "spreading" of the central bubble swarm. In the same time, with this closure, the liquid phase temperature decrease is lower in the centre of the tube. Considering thermal regime for hot water, the choice of the reference bubble diameter is found to influence greatly the prediction of the structure of the flow. The question is now to know what is the effect of such modelling for a more thermosenstive fluid.

In this work, the simulation of the cavitation of the refrigerant freon R114 in an expansion tube has been also investigated. As shown in the value of reference temperature variation $\Delta T^{*}$ in Tab. 4, this fluid is expected to be more thermosensitive than hot water. Results obtained for this fluid are reported in Fig. 8 at time $t=3.2 \mathrm{~ms}$ for the same reference diameters of $d_{b}=0.5-3-10 \mathrm{~mm}$ with initial conditions from Tab. 4. As shown in this figure, as for water, closure law considering inertial growth (red continuous line) gives again very similar results than considering an instantaneous mass transfer with velocity divergence model (Eq. 26, $\square$ ). When considering now a thermal growth regime (Eq. 24 and 14), the same behaviours are found on velocity, pressure $\left(p_{\text {sat } 0}=1.825\right.$ bar $)$, gas volume fraction and liquid phase temperature. As shown by Fig. 7, the maximal gas volume fraction in the center is decrease and a spreading of the central bubble swarm is also observed.

Finally, to appreciate operating temperature effect, for a given operating pressure and reference bubble diameter, some additional simulations, not reported here, with water $(T=290,320 \mathrm{~K})$ and $\mathrm{R} 114(\mathrm{~T}=230,260 \mathrm{~K}$ ) have been performed with this model based on a thermal regime closure law (Eq. 14, 11). It is found that the decrease of the operating temperature tend to decrease both the pressure level in the center of the tube and to sharpen the vapour production on both sides of the central gas pocket. This sharpens the resulting profiles generating larger local gradients of velocity and gas volume fraction. But in any case, a temperature decrease of a few degree is also found for the liquid phase in the center of the expansion tube is also found because at this location bubbles are trapped in the flow during the evaporation process. But in any case, in the center of the expansion tube, the liquid phase temperature is found to decrease of the same order of magnitude than with hot water $(1-2 K)$. However, in the literature, for the study of cavitating flows with cold water, it is usual to consider the isothermal hypothesis considering that in the cavitation area the temperature decrease of the liquid phase is insignificant. In fact, locally in cavitating cloud, the liquid phase temperature drop is influenced by phase change intensity but also by fluid velocity. 
Whatever the flow conditions, when vapor is produced (condensed), the liquid temperature should decrease (increase). But, in cavitating flow where the fluid velocity is high, such as over an hydrofoil, it is harder to measure a temperature decrease contrary to the case of cavitating flow where the fluid velocity is low, such as in the center of the tube of the present test case. Indeed even if the reference temperature drop of cold water $\Delta T^{*}=0.01 \mathrm{~K}$ is lower than the one of hot water $(0.27 \mathrm{~K})$ or R114 $(1.40 \mathrm{~K})$ this only means that, for a given Jakob number, the temperature difference between the liquid and gas phase would be lower than with those other fluids. In other words having a low $\Delta T^{*}$ do not insure of having a low temperature drop in the liquid phase of a cavitating flow. But having a very low (high) fluid velocity make easier (harder) the occurence and the measurement of liquid temperature variations induced by phase change.

\subsection{Phase change mechanism in the cavitating flow}

To understand how the thermal regime closure law affects simulation results we have plotted for the two considered test cases the evolution of the phasic temperature difference $\left(T_{L}-T_{G}\right)$, the density ratio $\left(\rho_{L} / \rho_{G}\right)$, the bubble Jakob and Nusselt numbers, and the ratio $a_{I} H_{L} / L$ as a function of the time for $x=0.5 \mathrm{~m}$ and for different bubble diameters. Results obtained for water are presented in Fig. 9. Globally, for all of bubble diameters considered, we can first notice that the different profiles decrease with the time. The intensity of phase change is stronger at the beginning of the simulation when temperature differences and density ratios are the largest. In the centre of the tube the development of velocity profile produces a decrease of the pressure. This decrease of pressure induces in fact a decrease of the gas phase temperature (Eq. 22) that quickly begins to be lower than the liquid phase temperature $\left(T_{G}<T_{L}\right)$ as well as a strong decrease of gas phase density that induces an increase of density ratio $\rho_{L} / \rho_{G}\left(\rho_{L 0} / \rho_{G 0}\left(H_{2} O\right) \approx 1900\right.$,

$\left.\rho_{L 0} / \rho_{G 0}(R 114) \approx 90\right)$. The temperature difference generates a heat flux toward the bubble surface that is used for phase change. In the same time the decrease of $\rho_{G}$ generates larger bubble volume expansion. Considering large bubble diameter $\left(d_{b}=10 \mathrm{~mm}\right)$, we get large Jakob and Nusselt numbers $\left(100<J a<10^{4}, 500<N u<3 \times 10^{4}\right)$ because the temperature difference and the density ratio are very important. Considering now small bubble $\left(d_{b}=0.5 \mathrm{~mm}\right)$, the temperature difference and density ratio are lower resulting in smaller Jakob $(6<J a<200)$ and Nusselt numbers $(40<N u<1000)$. However, in the same time with large bubbles the interfacial area $a_{I}=6 \alpha_{G} / d_{b}$ is lower than with smaller bubbles. As a result, the product $a_{I} H_{L} / L$ (Fig. 9d) is finally found to be smaller with large bubble than with small bubble and interfacial heat and mass transfers are lower resulting in larger temperature difference, Nusselt an Jakob numbers but also lower vapour production. Note that, the liquid phase temperature drop presented in Fig. 6d, 8d or $11 \mathrm{~d}$ results from the evaporation process occurring at bubbles interfaces. On the opposite, if we have considered a cavitating flow test case with condensation of bubbles, an increase of the liquid phase temperature would have been observed.

As shown in Fig. 10 for refrigerant R114, the same effect of increasing bubble reference diameter is found. This behaviour explained why for small reference bubble diameter the thermal regime closure law supplies very similar results than those considering instantaneous mass transfer. In addition, in comparison with models based on infinite relaxation parameter (or very small relaxation time) such the ones used by [71] or [14], the ratio $a_{I} H_{L} / L$ would in fact correspond to the relaxation parameter for phasic temperatures divided by the latent heat of evaporation : $\theta / L$ that in those approaches is considered to be infinite. As depicted in Fig. 9e and 10e, for the two 
considered cases, the ratio $a_{I} H_{L} / L$ is bounded between 3 and $200 \mathrm{~kg} \mathrm{~s}^{-1} \mathrm{~m}^{-2} \mathrm{~K}^{-1}$. As a consequence, considering the thermal growth regime is equivalent to admit that interfacial heat transfer, and thus phase change, can be finite rate in cavitating flows. Moreover, the bubble size is found to strongly affect the gas volume fraction distribution in the cavitating flow. One can also conclude from those original results that the size of bubbles in cavitating flow in thermal regime is thus very important for the estimation of the interfacial area through which heat and mass transfer takes place. Finally, it is very interesting to notice that using the proposed closure law, the variation of the gas density $\rho_{G}$ with pressure and temperature is also taken into account in the calculation of Jakob number since we have $J a \propto\left(\rho_{L} / \rho_{G}\right) \Delta T$. As a result, at a given position, a decrease of the gas phase density induces an increase of the Jakob number, Nusselt number and heat transfer coefficient. Our results show that, in contrast with common boiling flows, compressibility effects influence phase change kinetic through variation of $\rho_{L} / \rho_{G}$. In addition, it is very interesting to notice that for the case of bubble condensation due to a pressure increase, $\rho_{L} / \rho_{G}$ should decrease reducing Jakob number and interfacial heat and mass transfers.

\subsection{Influence of heat advection at the bubble surface}

In Fig. 7, 9 and 10, we have also reported results neglecting the effect of the Péclet number on the estimation of the Nusselt number (continuous red lines). To do that, the calculation of the Nusselt number was only based on the approximation of [64] so that $N_{u L}=N_{u L 0}$ (Eq. 11). As depicted in those figures, for present simulations, on one hand, it is found that for water the effect of the bubble Péclet number on the global solution can be neglected. On the other hand, for refrigerant R114, it is found that for large bubble the thermal Péclet number and thus the additional heat advection at the bubble surface has a small positive effect on the estimation of the transfer rates $a_{I} H_{L} / L$. This observation is mainly due to the fact that the Prandlt number in liquid R114 $(\mathrm{Pr}=4.8)$ is larger than in hot water $(\mathrm{Pr}=2.2)$ resulting in larger bubble Péclet number $\left(2400<\mathrm{Pe}(\mathrm{R} 144)<43400,800<\mathrm{Pe}\left(\mathrm{H}_{2} \mathrm{O}\right)<17000\right)$. To quantify this effect on the final solution we have compared the different profiles at the final time $t=3.2 \mathrm{~ms}$ for R114 at $|u|=2 \mathrm{~ms}^{-1}$ and $d_{b}=10 \mathrm{~mm}$. The differences are about $3 \%$ or less. This behaviour is manly due to the fact that Nusselt number is more sensitive to Jakob number variation with the time than to additional heat transfer due to bubble slip. As a result, for present simulations (flow conditions and fluid properties), at first order the Nusselt number can be estimated from Eq. 11.

\subsection{Influence of fluid properties variations on phase change kinetic}

For the simulation of phase change in boiling flows, it is usual to consider a constant latent heat and a constant liquid phase conductivity [53, 57, 68, 66]. For cavitating flow in thermal regime, this point is still an open question. In results presented above, the Jakob number $J a=$ $\rho_{L} C p_{L} \Delta T /\left(\rho_{G} L\right)$ is calculated with variable $\rho_{L}, \rho_{G}, \Delta T$ that are evaluated during the resolution of equations while heat capacity $C_{p L}$ and vaporisation latent heat $L$ are considered to be constant. In addition, heat transfer coefficient and Péclet number are calculated with a constant conductivity $\lambda_{L}$. An analysis of the variation of fluid properties of water for $273<T<355 K$ and of R114 for $273<T<293 K$ shows us that the variation of the constant pressure heat capacity of the liquid with the temperature is less than $3 \%$ so that it does not influence the estimation of Jakob numbers. However, a strongest dependency on the temperature is found in the estimation of the liquid phase conductivity $\lambda_{L}$ (15\% for $\mathrm{H}_{2} \mathrm{O}, 7 \%$ for R114) and the latent heat of evaporation $L$ ( $8 \%$ for $\mathrm{H}_{2} \mathrm{O}, 5 \%$ for R114). The question is thus to know if taking into account the variation of latent 
heat and liquid phase conductivity influences the result. Correlation used to describe the variation of $L$ and $\lambda_{L}$ with the temperature are based on data given by [79] at the saturation temperature. The temperature that is considered for the calculation of the variation of $L$ and $\lambda_{L}$ is taken as the temperature at the bubble surface that corresponds to the gas phase temperature $T_{G}$. For simulation with water at $|u|=2 \mathrm{~m} / \mathrm{s}$ with $d_{b}=10 \mathrm{~mm}$, some small differences can be denoted. Compared to constant $L$ and $\lambda_{L}$ case, we get lower pressure $(-15 \%)$ and lower gas volume fraction $(-6 \%)$ in the centre of the tube. For simulation with R114 at $|u|=2 \mathrm{~m} / \mathrm{s}$ with $d_{b}=10 \mathrm{~mm}$, the same behaviour is found with a small decrease of pressure $(-4 \%)$ and gas volume fraction $(-6 \%)$ in the centre of the tube. Consequently, at first order, the calculation of the Jakob number and heat transfer induced by phase change can be performed with constant $C_{p L}, L$ and $\lambda_{L}$ while phasic density $\left(p_{L}, p_{G}\right)$ and temperature difference $(\Delta T)$ are given by solving mixture model equations. Consequently, taking into account the variation of latent heat $L$ and heat conduction of the liquid phase $\lambda_{L}$ with the temperature can improve the accuracy of calculations. This can be a perspective of a future work.

\subsection{Simulation at larger stretching velocity}

We consider here the cavitation of hot water at $T=355 \mathrm{~K}$ with a stretching velocity of $|u|=50 \mathrm{~ms}^{-1}$. The resident time of bubble is now $\tau_{\text {res }}=l / u \approx 0.02 \mathrm{~s}$. With a transition time of $\tau \approx 10^{-7}-10^{-4} s$ (Fig. 3), the condition $\tau / \tau_{\text {res }}<<1$ is also verified for that case and thermal growth closure law can be employed. Velocity, pressure, gas volume fraction and liquid phase temperature profiles obtained for this case are displayed in Fig. 11 for the time $t=2.5 \mathrm{~ms}$ and reference bubble diameters of $d_{b}=0.5,1,3 \mathrm{~mm}$. One can note that, since inertial growth source term (Eq. 25) is much larger than the one based on thermal growth (Eq. 23), it generates easily oscillation of the solution. For this reason, it was only possible to use it at low stretching velocity. We will thus compare our results with the one provided by the velocity divergence model (Eq. 26). As previously, for $d_{b}=0.5 \mathrm{~mm}$, the solution obtained using the thermal regime closure is found to be close to the one predicted by Eq. 26 corresponding to instantaneous mass transfer. As for cases at lower stretching velocity, the development of the velocity profile generates a pressure decrease that induces phase change by decreasing gas phase temperature below the liquid phase temperature. For such rude conditions, the model predicts that, along a large part at the centre of the tube for $0.4<x<0.6 \mathrm{~m}$, the velocity is almost cancelled while the gas volume fraction reaches unity $\alpha_{G}=1$. The induced vapour production generates a strong decrease of liquid phase temperature $(30-50 \mathrm{~K})$. As depicted in Fig. 11a and c, for a large stretching velocity the effect of a change in reference bubble diameter as a weak effect on the profile of gas volume fraction and velocity. However, variation of the bubble diameter seems to have more effect on the pressure and temperature profiles.

For such cases where the gas volume fraction reaches locally its maximal value $\left(\alpha_{G}=1\right)$ on a large part of the flow, the hypothesis on the structure of the gas-liquid flow as "a dispersion of bubbles" begins to be questionable. Indeed, if the gas-liquid bubbly flow transforms into a large gas pocket in the centre of the tube the calculation of the interfacial area $a_{I}=6 \alpha_{G} / d_{b}$ and the closure on the Nusselt number to describe phase change need to be modified. For a large gas pocket, such those observed in super cavitating flows [80], the interfacial area strongly decreases

and the heat transfer is also drastically different. But this point is out of the scope of the present study that consider cavitation as a dispersed bubbly flow. 


\section{Conclusions}

In this work, we have first presented the two main bubble growth regimes that we can found in bubbly flows with phase change: inertial and thermal growth. Thanks to the calculation of the time $\tau$ corresponding to the inception of the thermal regime and the end of inertial regime, we have shown that thermal controlled growth is very likely to occur in the majority of the cavitating flow with thermosensitive liquids if the resident time of bubbles in the low pressure area is large enough $\left(\tau / \tau_{\text {res }}<<1\right)$. Surprisingly, the time of transition between the inertial and thermal regime is found to be only dependant on the fluid physical properties.

To evaluate the effect of considering a phase change limited by interfacial heat transfer at bubble surface, different simulations of cavitation in expansion tubes were performed with hot water and refrigerant R114 using a four-equations mixture model with Euler formulation. For those fluids and operating temperatures, a comparison of heat diffusivity and conductivity has demonstrated that temperature in the gas phase can be considered as constant and equal to the saturation temperature that is calculated here from the local pressure using the Antoine's equation. The model studied in this work is based on the Nusselt number calculating from Jakob and thermal Péclet numbers in order to take into account bubble growth due to phase change and also bubble relative velocity effect on bubble growth. This approach is based on recent works dedicated to phase change modelling in bubbly flows.

Numerical simulations of cavitation with mass transfer at moderate stretching velocity have been performed and compared to solutions obtained with mass transfer based on inertial growth and instantaneous mass transfer models (infinite relaxation parameter or velocity divergence model). It is found that model considering instantaneous mass transfer gives results very similar to those obtained with mass transfer considering inertial regime. This is explained because interfacial velocities predicted by inertial growth are so important that mass transfer can be considered as instantaneous. In the opposite, considering thermal growth gives finite rate heat and mass transfer terms that strongly depends on Jakob number and reference bubble diameter (interfacial area). When considering a thermal growth, the local maxima of gas volume fraction and the minimum pressure in the flow are decreased. But indeed, when decreasing the reference bubble diameter, the solution obtained in thermal regime match the one given by instantaneous mass transfer model. In addition, for present simulations, fluids and flow conditions, at first order the Nusselt number can be estimated as a function of the Jakob number using the approximation proposed by [64]. It is also shown that, in the calculation of the Jakob number, the latent heat and liquid phase conductivity can be considered as constant so that Jakob number variation is monitored by phasic temperature difference and density ratio variation so that $J a \propto\left(\rho_{L} / \rho_{G}\right) \Delta T$. As a consequence, for a local pressure reduction, compressibility effects promote vapour production because both density ratio and Jakob number increase. On the opposite, for a local pressure increase, compressibility effects should slow down vapour condensation because both density ratio and Jakob number decrease. Finally, some tests Moreover, simulations at large stretching velocity seem to show us that the influence of bubble diameter on the solution is less important. However, for such cases, the limit of the proposed closure law is reached since it results in the formation of a large central gas pocket that can no longer be considered as a bubble dispersion characterised by a large interfacial area. Finally, some tests on the effect of the description of fluid properties tend to show that taking into account the variation of latent heat and liquid phase conduction with the temperature can affect the result of around $4-15 \%$. 
The first perspective of this work is to complete this description of cavitation with a model for vapour bubble condensation. Indeed, in this work, we have focus on bubble growth but the physical description of bubble collapse due to vapour condensation is also a very important issue. The second perspective is to implement this model based on thermal growth with a viscous formulation and to test it on different applications such as venturis, diaphragms, pumps or turbopump inducers. The third perspective is also to deepen the study of model behaviour respect to the influence of fluid properties variations with the temperature. An other perspective, is also to take into account the effect of dissolved gas in the liquid phase on the intensity of cavitation phenomena.

\section{Acknowledgements}

The authors would like to thank the CNES, French space agency, for supporting this work and especially J. Dehouve, S. Palerm and J. Herpe. The laboratory LEGI is part of the LabEx Tec 21 (Investissements d'Avenir - grant agreement ANR-11-LABX-0030).

\section{References}

[1] Y. Delannoy, J. Kueny, Two phase flow approach in unsteady cavitation modeling, Cavitation and Multiphase Flow Forum, ASME-FED 98 (1990) 153-158.

[2] R. Saurel, R. Abgrall, A multiphase godunov method for compressible multifluid and multiphase flows, J. Comput. Phys. 150 (1999) 425-467.

[3] E. Goncalvès, Numerical study of expansion tube problems: Toward the simulation of cavitation, Comput. Fluids 72 (2013) 1-19.

[4] G. Schnerr, J. Sauer, Physical and numerical modeling of unsteady cavitation dynamics, 4th International Conference on Multiphase Flow, New Orleans, USA.

[5] S. Mimouni, M. Boucker, J. Laviéville, A. Guelfi, D. Bestion, Modelling and computation of cavitation and boiling bubbly flows with the neptune cfd code, Nuclear Engineering and Design 238 (2008) 680-692.

[6] E. Goncalves, R. Fortes Patella, J. Rolland, B. Pouffary, G. Challier, Thermodynamic effect on a cavitating inducer in liquid hydrogen, J. Fluids Eng. 132 (11) (2010) 111305.

[7] O. Coutier-Delgosha, J. Reboud, Y. Delannoy, Numerical simulation of the unsteady behaviour of cavitating flows, Int. J. Numer. Methods Fluids 42 (5) (2003) 527-548.

[8] R. Fortes-Patella, S. Barre, J. Reboud, Experiments and modelling of cavitationg flows in venturi, part ii : Unsteady cavitation, Sixth International Symposium on Cavitation CAV2006, Wageningen, The Netherlands, September.

[9] E. Sinibaldi, F. Beux, M. Salvetti, A numerical method for 3d barotropic flows in turbomachinery, Flow Turbulence Combust. 76 (2006) 371-381.

[10] B. Pouffary, R. Fortes Patella, J. L. Reboud, P. Lambert, Numerical analysis of cavitation instabilities in inducer blade cascade, J. Fluids Eng. 130 (4) (2008) 041302-41308. 
[11] N. Gonzalo Flores, E. Goncalves Da Silva, R. Fortes Patella, J. Rolland, C. Rebattet, Head drop of a spatial turbopump inducer, J. Fluids Eng. 131 (11) (2008) 111301.

[12] H. Jese, R. Fortes-Patella, S. Antheaume, High head pump-turbine: Pumping mode numerical simulations with a cavitation model for off-design conditions, IOP Conf. Series: Earth and Environmental Science 22 (2013) 032048.

[13] J. Moreau, O. Simonin, C. Habchi, A numerical study of cavitation influence on diesel jet atomisation, 19th Annual Meeting of the Institute for Liquid Atomization and Spray Systems, Nottingham, 6-8 September.

[14] A. Zein, M. Hantke, G. Warnecke, Modeling phase transition for compressible two-phase fows applied to metastable liquids, J. Comput. Phys. 229 (2010) 2964-98.

[15] M. Baer, J. Nunziato, A two-phase mixture theory for the deflagration-to-detonation transition in reactive granular materials, Int. J. Multiphase Flow 12 (1986) 861-889.

[16] E. Goncalvès, Modeling for non isothermal cavitation using 4-equation models, Int. J. Heat Mass Transfer 76 (2014) $247-262$.

[17] L. Rayleigh, On the pressure developed in a liquid during the collapse of a spherical cavity, Philosophical Magazine Series 634 (200) (1917) 94-98.

[18] A. Kubota, H. Kato, H. Yamaguchi, A new modelling of cavitating flows: a numerical study of unsteady cavitation on a hydrofoil section, J. Fluid Mech. 240 (1992) 59-96.

[19] A. Singhal, M. Athavale, H. Li, Y. Jiang, Mathematical basis and validation of the full cavitation model, J. Fluids Eng. 124 (2002) 617.

[20] P. Zwart, A. Gerber, T. Belamri, A two-phase flow model for predicting cavitation dynamics, ICMF 2004 International Conference on Multiphase Flow, Yokohama, Japan, May 30-June $3,2004$.

[21] C. Merkle, J. Feng, P. Buelow, Computational modeling of the dynamics of sheet cavitation, Third International Symposium on Cavitation, Grenoble, France, April.

[22] R. Kunz, D. Boger, D. Stinebring, T. Chyczewski, J. Lindau, H. Gibeling, S. Venkateswaran, T. Govindan, A preconditioned navier-stokes method for two-phase fows with application to cavitation prediction, Comput. Fluids 29 (8) (2000) 849-875.

[23] M. Morgut, E. Nobile, I. Bilus, Comparison of mass transfer models for the numerical prediction of sheet cavitation around a hydrofoil, Int. J. Multiphase Flow 37 (2011) 620-626.

[24] Y. Ait Bouziad, M. Farhat, F. Guennoun, J. Kueny, F. Avellan, K. Miyagawa, Physical modelling and simulation of leading edge cavitation, application to an industrial inducer, Fifth International Symposium on Cavitation (CAV2003), Osaka, Japan, November 1-4.

[25] S. Yakubov, B. Cankurt, P. Schiller, M. Abdel-Maksoud, T. Rung, An advanced eulerlagrange approach to numerical simulation of cavitating engeneering flows, Proceedings of the Eighth International Symposium on Cavitation (CAV 2012), 
[26] R. Medvitz, R. Kunz, D. Boger, J. Lindau, A. Yocum, Performance analysis of cavitating flow in centrifugal pumps using multiphase cfd, Proceedings of ASME Fluids Engineering Conference, May 29 - June 1, New Orleans, Louisiana.

[27] M. Frobenius, R. Schilling, J. Friedrichs, G. Kosyna, Numerical and experimental investigation of cavitating flow in a centrifugal pump impeller, ASME Fluid Engineering Division Summer Meeting, Montreal 2002.

[28] R. Kunz, J. Lindau, T. Kaday, L. Peltier, A numerical method for 3d barotropic flows in turbomachinery, Fifth International Symposium on Cavitation (CAV2003), Osaka, Japan, November 1-4.

[29] M. Dular, R. Bachert, B. Stoffel, B. Sirok, Experimental evaluation of numerical simulation of cavitating flow around hydrofoil, Eur. J. Mech. B Fluids 24 (2005) 522-538.

[30] D. Li, M. Grekula, P. Lindell, Towards numerical prediction of unsteady sheet cavitation on hydrofoils, 9th International Conference on Hydrodynamics, October 11-15, Shanghai, China (2010) 699.

[31] C. Vortmann, G. Schnerr, S. Seelecke, Thermodynamic modeling and simulation of cavitating nozzle flow, Int. J. Heat Fluid Flow 24 (2003) 774-783.

[32] A. Hosangadi, V. Ahuja, Numerical study of cavitation in cryogenic fluids, J. Fluids Eng. 127-281 (2005) 267.

[33] Y. Utturkar, J. Wu, G. Wang, W. Shyy, Recent progress in modeling of cryogenic cavitation for liquid rocket propulsion, Prog. Aerosp. Sci. 41 (2005) 558-608.

[34] E. Rapposelli, L. d Agostino, A barotropic cavitation model with thermodynamic effets, Fifth International Symposium on Cavitation (cav2003), Osaka, Japan, November 1-4.

[35] N. Tani, S. Tsuda, N. Yamanishi, Y. Yoshida, Development and validation of new cryogenic cavitation model for rocket turbopump inducer, Proceedings of the 7th International Symposium on Cavitation, August 17-22, 2009, Ann Arbor, Michigan, USA.

[36] C. Tseng, W. Shyy, Modeling for isothermal and cryogenic cavitation, Int. J. Heat Mass Transfer 53 (2010) 513-525.

[37] J. Sauer, G. Winkler, G. Schnerr, Cavitation and condensation - common aspects of physical modeling and numerical approach, Chem. Eng. Technol. 23 (8) (2000) 663.

[38] R. Saurel, O. Lemetayer, A multiphase model for compressible flows with interfaces, shocks, detonation waves and cavitation, J. Fluid Mech. 431 (2001) 239-271.

[39] S. Mimouni, A. Archer, J. Laviéville, M. Boucker, N. Méchitoua, Modélisation et simulation des écoulements cavitants par une approche diphasique, La Houille Blanche 6 (2006) 121128.

[40] J. Hord, Cavitation in liquid cryogens ii - hydrofoil, NASA CR-2156. 
[41] J. Hord, Cavitation in liquid cryogens iii - ogives, NASA CR-2242.

[42] J. Holl, M. Billet, D. Weir, Thermodynamic effects on developed cavitation, ASME, Transactions, Series I - J. Fluids Eng. 97 (1975) 507-513.

[43] D. Fruman, J. Reboud, B. Stutz, Estimation of thermal effects in cavitation of thermosensible liquids, Int. J. Heat Mass Transfer 42 (1999) 3195-3204.

[44] J. Franc, C. Rebattet, A. Coulon, An experimental investigation of thermal effects in a cavitating inducer, Transactions of the ASME 126 (2004) 716-722.

[45] Y. Yoshida, K. Kikuta, S. Hasegawa, M. Shimagaki, T. Tokumasu, Thermodynamic effect on a cavitating inducer in liquid nitrogen, J. Fluids Eng. 129 (2007) 273-278.

[46] J. Franc, G. Boitel, M. Riondet, E. Janson, P. Ramina, C. Rebattet, Thermodynamic effect on a cavitating inducer - part i: Geometrical similarity of leading edge cavities and cavitation instabilities, J. Fluids Eng. 132 (2010) 021303-1.

[47] F. Ayela, M. Medrano-Muñoz, D. Amans, C. Dujardin, T. Brichart, M. Martini, O. Tillement, G. Ledoux, Experimental evidence of temperature gradients in cavitating microflows seeded with thermosensitive nanoprobes, Phys. Rev. E 88 (2013) 043016.

[48] F. Ayela, D. Colombet, G. Ledoux, O. Tillement, Cartographie thermique au sein d'ecoulements cavitants, La Houille Blanche 1 (2015) 103-108.

[49] M. Petkovsec, M. Dular, Ir measurements of the thermodynamic effects in cavitating flow, Int. J. Heat Fluid Fl. 44 (2013) 756-763.

[50] H. Stahl, J. Stepanoff, N. Phillipsburg, Thermodynamic aspects of cavitation in centrifugal pumps, Transactions of the ASME Journal of basic engineering 78 (1956) 1691-1693.

[51] A. Stepanoff, Cavitation properties of liquids, Journal of Engineering for Power (1964) 195200.

[52] M. Rodio, M. De Giorgi, A. Ficarella, Influence of convective heat transfer modeling on the estimation of thermal effects in cryogenic cavitating flows, Int. J. Heat Mass Transfer 55 (2012) 6538-6554.

[53] L. Florschuetz, B. Chao, On the mechanics of vapour bubble collapse, Trans. ASME, J. Heat Transfer 87 (209).

[54] M. Plesset, A. Prosperetti, Bubble dynamic and cavitation, Annu. Rev. Fluid Mech. 9 (1977) 145-185.

[55] C. Brennen, Cavitation and bubble dynamics, Oxford engineering science series 44, 1995.

[56] D. Legendre, Quelques aspects des forces hydrodynamiques et des transferts de chaleur sur une bulle sphérique, Ph.D. thesis, INP Toulouse (1996). 
[57] D. Legendre, J. Borée, J. Magnaudet, Thermal and dynamic evolution of a spherical bubble moving steadily in a superheated or subcooled liquid, Phys. Fluids 10 (6) (1998) 1256-1272.

[58] M. Plesset, The dynamics of cavitation bubbles, J. Appl. Mechanics 16 (1949) 277-282.

[59] M. Ishii, Thermo-fluid dynamic theory of two-phase flow, Eyrolles, 1975.

[60] L. Scriven, On the dynamic of phase growth, Chem. Eng. Sci. 10 (1-2) (1959) 1-13.

[61] J. Bonnin, Thermodynamic effects in cavitation, Inst. of Mech. Eng. Conference on Cavitation.

[62] J. Franc, E. Janson, P. Ramina, G. Boitel, M. Riondet, C. Rebattet, Thermodynamic effect on a cavitating inducer - part ii: On-board measurements of temperature depression within leading edge cavities, J. Fluids Eng. 132 (2010) 021304.

[63] M. Plesset, S. Zwick, The growth of vapor bubbles in superheated liquids, J. Appl. Phys. 25 (4) (1954) 493-500.

[64] D. Labuntsov, B. Kol'chugin, V. Golovin, E. Zakharova, L. Vladimirova, Investigation of bubble growth in boiling saturated water by high-speed photography for a wide range of variation of the pressure, Teplofizika Vysokikh Temperatur 2 (3) (1964) 446-453.

[65] E. Rückenstein, On heat transfer between vapour bubbles in motion and the boiling liquid from which they are generated, Chem. Eng. Sci. 10 (1959) 22-30.

[66] S. Tanguy, S. Sagan, B. Lalanne, F. Couderc, C. Colin, Benchmarks and numerical methods for the simulation of boiling flows, J. Comput. Phys. 264 (2014) 1-22.

[67] V. Levich, Physicochemical hydrodynamics, Prentice-Hall, Englewood Cliffs, NJ, 1972.

[68] P. Oresta, R. Verzicco, D. Lohse, A. Prosperetti, Heat transfer mechanisms in bubbly rayleighbénard convection, Phys. Rev. E 80 (2009) 026304.

[69] M. Ishii, T. Hibiki, Thermo-Fluid Dynamics of Two-Phase Flow, Springer, 2006.

[70] A. Murrone, H. Guillard, A five equation reduced model for compressible two phase flow problems, J. Comput. Phys. 202 (2005) 664-698.

[71] F. Petitpas, E. Franquet, R. Saurel, O. Le Metayer, A relaxation-projection method for compressible flows. part ii: Artificial heat exchanges for multiphase shocks, J. Comput. Phys. 225 (2007) 2214-2248.

[72] A. Kapila, R. Menikoff, J. Bdzil, S. Son, D. Stewart, Two-phase modeling of deflagration-todetonation transition in granular materials: Reduced equations, Phys. Fluids 13 (10) (2001) 3002 .

[73] R. Saurel, F. Petitpas, R. Abgrall, Modelling phase transition in metastable liquids: application to cavitating and flashing flows, J. Fluid Mech. 607 (2008) 313-350. 
[74] C. Antoine, Tensions des vapeurs; nouvelle relation entre les tensions et les temperatures, Comptes Rendus des Seances de l'Academie des Sciences 107 (1888) 681-684.

[75] W. Dijkhuizen, I. Roghair, M. Van Sint Annaland, J. Kuipers, Dns of gas bubbles behaviour using an improved $3 \mathrm{~d}$ front tracking model-drag force on isolated bubbles and comparison with experiments, Chem. Eng. Sci. 65 (4) (2010) 1415-1426.

[76] A. Wood, A Textbook of Sound, G. Bell and Sons Ltd, London, 1930.

[77] G. Wallis, One-dimensional Two-phase Flow, McGraw-Hill, New-York, 1969.

[78] V. Rusanov, Calculation of interaction of non-steady shock waves with obstacles, J. Comput. Math Phys. 1 (1961) 267-79.

[79] E. Lemmon, M. McLinden, D. Friend, Thermophysical Properties of Fluid Systems in NIST Chemistry WebBook, NIST Standard Reference Database Number 69, Eds. P.J. Linstrom and W.G. Mallard, National Institute of Standards and Technology, Gaithersburg MD, 20899, http://webbook.nist.gov, 2012.

[80] J. Franc, J. Michel, Attached cavitation and the boundary layer: experimental investigation and numerical treatment, J. Fluid Mech. 154 (1985) 63-90.

[81] C. Yaws, Thermophysical properties of chemicals and hydrocarbons, William Andrew Inc., 2008.

[82] E. Sanjari, M. Honarmand, H. Badihi, A. Ghaheri, An accurate generalized model for predict vapor pressure of refrigerants, Int. J. Refrig. 36 (4) (2013) 1327-1332. 


\section{Nomenclature}

\section{List of symbols}

$a_{I} \quad$ volumetric interfacial area, $m^{-1}$

$B \quad$ B factor

$C_{p k} \quad$ specific heat capacity of phase $k$ at constant pressure, $J k^{-1} K^{-1}$

$C_{v k} \quad$ specific heat capacity of phase $k$ at constant volume, $J k^{-1} K^{-1}$

$c_{w} \quad$ Wood's speed of sound, $m s^{-1}$

$c_{E O S} \quad$ speed of sound based on sinus equation of state, $\mathrm{ms}^{-1}$

$c_{k} \quad$ speed of sound of phase $k, \mathrm{~m} \mathrm{~s}^{-1}$

$d_{b} \quad$ reference mean Sauter diameter of the bubble size distribution, $m$

$D_{k}^{t h} \quad$ thermal diffusivity of phase $k, D_{k}^{t h}=\lambda_{k} /\left(\rho_{k} C_{p k}\right)$

$e_{k} \quad$ specific internal energie of phase $k, \mathrm{~J} \mathrm{~kg}^{-1}$

$E_{k} \quad$ total energy of phase $k, J k^{-1}$

$E_{m} \quad$ gas liquid mixture total energy, $\mathrm{J} \mathrm{kg}^{-1}$

$H_{L} \quad$ heat transfer coefficient in phase $k, W m^{-2} K^{-1}$

$h_{k} \quad$ specific enthalpie of phase $k, \mathrm{~J} \mathrm{~kg}^{-1}$

$J_{l o c} \quad$ local mass flux, $\mathrm{kg} \mathrm{m}^{-2} \mathrm{~s}^{-1}$

$J a \quad$ Jakob number, $J a=\rho_{L} C_{p L} \Delta T /\left(\rho_{G} L\right)$

$l \quad$ mean length of bubble path in the low pressure area where $p<p^{\text {sat }}, m$

$L \quad$ latent heat of vaporisation $L=h_{G}-h_{L}, J k^{-1}$

$N_{u L} \quad$ Nusselt number

$N_{u L 0} \quad$ Nusselt number wihout wlip

$p \quad$ pressure, $\mathrm{Pa}$

$p_{k}^{\infty} \quad$ pressure reference in stiffened gas equation of state, $P a$

$P e \quad$ thermal Péclet number, $P e=U d_{b} / D_{L}^{t h}$

$P e_{c} \quad$ critical thermal Péclet number,

$\operatorname{Pr} \quad$ Prandtl number, $\operatorname{Pr}=\nu_{L} / D_{L^{t h}}$

$q_{k}^{\prime \prime} \quad$ interfacial heat flux from phase $k, J m^{-3} s^{-1}$

$R \quad$ bubble radius, $m$

Re bubble Reynolds number, $R e=\rho_{L} v_{r} d_{b} / \mu_{L}$

$s \quad$ surface tension of the fluid, $\mathrm{Nm}^{-1}$

$S_{b} \quad$ bubble surface, $m^{2}$

$t \quad$ time, $s$

$T_{k} \quad$ tempreature of phase $k, K$

$u \quad$ stretching velocity, $m s^{-1}$

$V_{b} \quad$ bubble volume, $m^{3}$

$v_{k} \quad$ velocity of phase $k, \mathrm{~ms}^{-1}$

$v_{m} \quad$ gas liquid mixture velocity, $m s^{-1}$

$v_{r} \quad$ mean relative velocity between phases, $v_{r}=v_{G}-v_{L}, \mathrm{~ms}^{-1}$

\section{Greek symbols}

$\alpha_{k} \quad$ volume fraction of phase $k$

$\gamma_{k} \quad$ heat capacity ratio for phase $k, \gamma_{k}=C_{p k} / C_{v k}$

$\Gamma_{k} \quad$ mass transfer term of phase $k, \mathrm{~kg} \mathrm{~m}^{-3} \mathrm{~s}^{-1}$ 


$\begin{array}{ll}\lambda_{k} & \text { conductivity of phase } k, W \mathrm{~m}^{-1} \mathrm{~K}^{-1} \\ \mu_{k} & \text { dynamic viscosity of phase } k, P a s \\ \nu_{k} & \text { kinematic viscosity of phase } k, \mathrm{~m}^{2} \mathrm{~s}^{-1} \\ \pi_{k} & \text { internal energy reference in stiffened gas equation of state, } \mathrm{J} \mathrm{kg}^{-1} \\ \rho_{k} & \text { density of phase } k, \mathrm{~kg} \mathrm{~m}^{-3} \\ \tau & \text { time for inception of thermal regime, } \mathrm{s} \\ \tau^{\prime} & \text { time for inception of thermal regime with effect of the relative velocity, } \mathrm{s} \\ \tau_{\text {res }} & \text { resident time of bubbles in the low pressure area, } \mathrm{s}\end{array}$

\section{Supercripts}

I at the bubble surface

sat at saturation

$\mathrm{T} \quad$ at triple point

\section{Subscripts}

$\mathrm{G} \quad$ gas phase

$\mathrm{k} \quad$ phase $k$

L liquid phase

$\mathrm{m}$ gas liquid mixture

0 initial value 


\begin{tabular}{l|ccc}
\hline & $T$ & $\lambda_{L} / \lambda_{G}$ & $D_{G}^{t h} / D_{L}^{t h}$ \\
& $K$ & - & - \\
\hline water & 293 & 30 & 1000 \\
water & 355 & 30 & 200 \\
$R 114$ & 293 & 5 & 20 \\
$N_{2}$ & 78 & 20 & 10 \\
$O_{2}$ & 90 & 20 & 20 \\
$H_{2}$ & 23 & 5 & 4 \\
\hline
\end{tabular}

Table 1: Thermal conductivity ratio and thermal diffusion coefficient ratio for different common cavitating fluids.

\begin{tabular}{c|cccc}
\hline & $p_{L}^{\infty}$ & $p_{G}^{\infty}$ & $\pi_{L}$ & $\pi_{G}$ \\
& $P a$ & $P a$ & $\mathrm{~J} \mathrm{~kg}^{-1}$ & $\mathrm{~J} \mathrm{~kg}^{-1}$ \\
\hline water & $10^{9}$ & 0 & -1167000 & 2030000 \\
R114 & $1.21 \times 10^{8}$ & 0 & -69000 & 144240 \\
\hline
\end{tabular}

Table 2: Parameters used for stiffened gas equation of state.

\begin{tabular}{c|ccc}
\hline & $\mathrm{A}$ & $\mathrm{B}$ & $\mathrm{C}$ \\
& - & $K$ & $K$ \\
\hline water & 8.05573 & 1723.6425 & 233.08 \\
R114 & 6.871 & 942.3 & 232.6 \\
\hline
\end{tabular}

Table 3: Parameters used for Antoine's equation according to [81] and [82]. 


\begin{tabular}{|c|c|c|c|c|c|c|c|c|}
\hline & $\begin{array}{l}T_{0} \\
K\end{array}$ & $\begin{array}{l}p_{0} \\
\text { bar }\end{array}$ & $\begin{array}{c}\rho_{L} \\
k g g m^{-3}\end{array}$ & $\begin{array}{c}\rho_{G} \\
\mathrm{kgm}^{-3}\end{array}$ & $\begin{array}{c}\mu_{L} \\
\text { Pas }\end{array}$ & $\begin{array}{c}\lambda_{L} \\
W(m K)^{-1}\end{array}$ & $\begin{array}{c}C_{p L} \\
J(k g K)^{-1}\end{array}$ & $\begin{array}{c}C_{p G} \\
J(k g K)^{-1}\end{array}$ \\
\hline water & 355 & 1 & 1149.9 & 0.59 & 0.0003 & 0.67 & 4267 & 1487 \\
\hline \multirow[t]{4}{*}{ R114 } & 293 & 3 & 1470.3 & 15.64 & 0.0003 & 0.062 & 984 & 700 \\
\hline & & $\begin{array}{c}\gamma_{L} \\
-\end{array}$ & $\gamma_{G}$ & $\begin{array}{c}L \\
k J k g^{-1}\end{array}$ & $\begin{array}{l}p^{\text {sat }} \\
\text { bar }\end{array}$ & $\begin{array}{c}d T^{s a t} / d p \\
K b a r^{-1}\end{array}$ & $\begin{array}{c}\Delta T^{*} \\
K\end{array}$ & $\begin{array}{l}p^{T} \\
P a\end{array}$ \\
\hline & water & 2.35 & 1.43 & 2304 & 0.51 & 50 & 0.27 & 611 \\
\hline & R114 & 1.40 & 1.10 & 130 & 1.825 & 21 & 1.40 & 236 \\
\hline
\end{tabular}

Table 4: Initial conditions and physical properties considered at saturation for $T=T_{0}$. 


\begin{tabular}{l|cccccccccc}
\hline nb. of cells & 100 & 200 & 400 & 800 & 2000 & 3000 & 4000 & 5000 & 6000 & 8000 \\
\hline water - case 1 & 0.439 & 0.434 & 0.429 & 0.426 & 0.425 & 0.424 & 0.424 & 0.424 & 0.424 & 0.424 \\
R114 - case 2 & 0.758 & 0.727 & 0.706 & 0.689 & 0.673 & 0.670 & 0.669 & 0.668 & 0.667 & 0.666 \\
water - case 3 & 0.199 & 0.160 & 0.140 & 0.131 & 0.117 & 0.107 & 0.099 & 0.092 & 0.087 & 0.087 \\
\hline
\end{tabular}

Table 5: Minimum value of pressure obtained in the expansion tube for different grid spacing (case $1:|u|=2 \mathrm{~ms}{ }^{-1}$, $d_{b}=1 \mathrm{~mm}, t=3.2 \mathrm{~ms}$; case $2:|u|=2 \mathrm{~ms}^{-1}, d_{b}=3 \mathrm{~mm}, t=3.2 \mathrm{~ms}$; case $3:|u|=50 \mathrm{~ms}^{-1}, d_{b}=1 \mathrm{~mm}$, $t=2.5 \mathrm{~ms})$. 


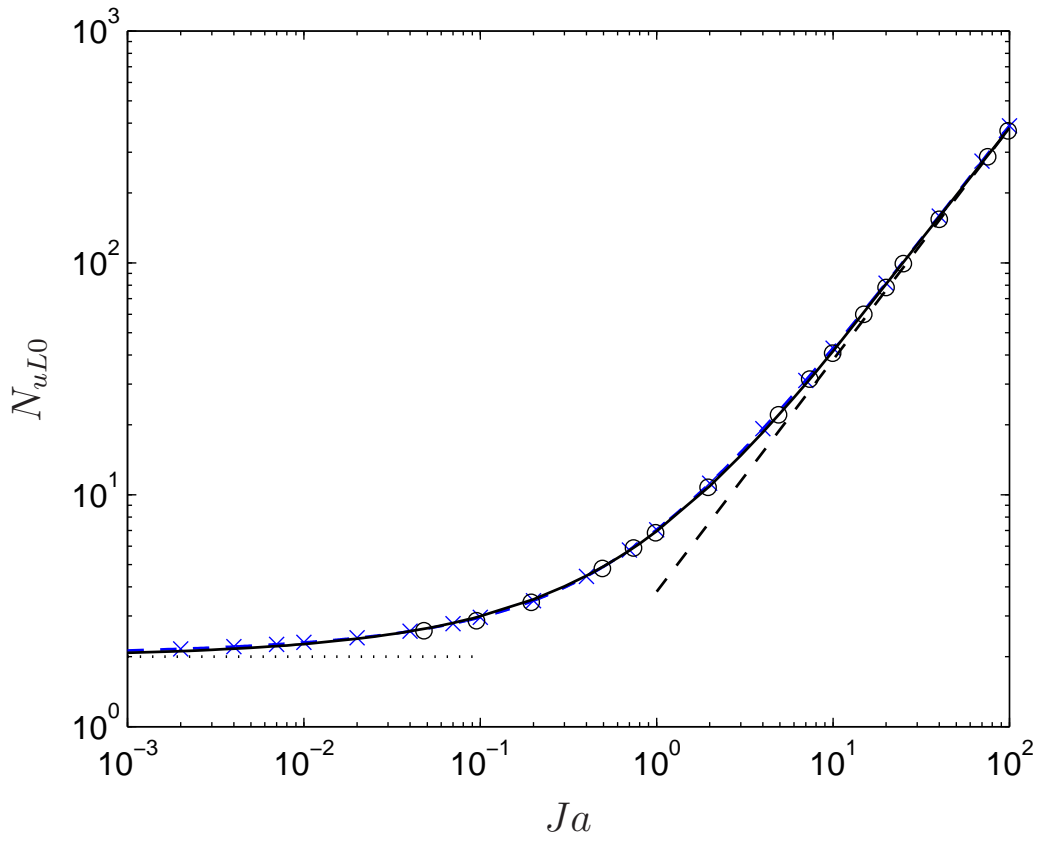

Figure 1: Nusselt number $N_{u L 0}$ as a function of Jakob number for bubble thermal growth with no slip between the bubble and the liquid phase: - [60] (Eq. 8, 9, 10), .. $N_{u L 0}(J a \rightarrow 0)=2,--[63] N_{u L 0}(J a \rightarrow \infty)=12 J a / \pi$, 。 direct numerical simulations of [57], $-\times$ [64] Eq. 11. 


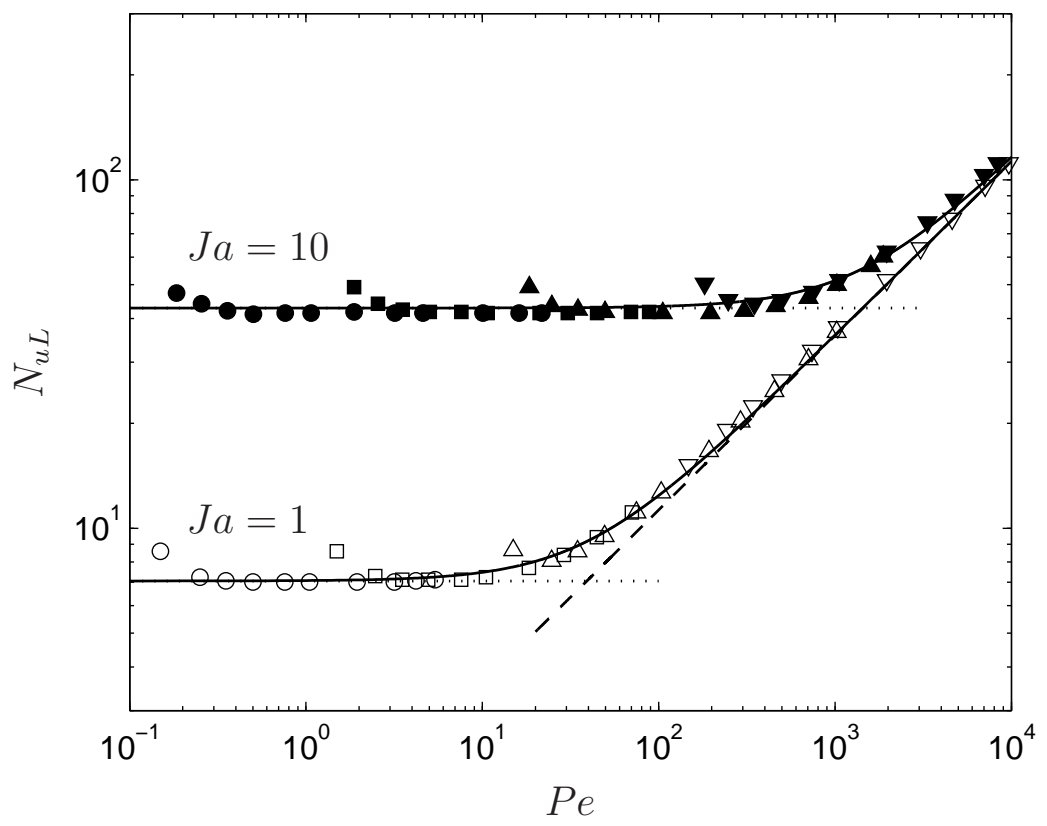

Figure 2: Nusselt number $N_{u L}$ as a function of Péclet number for bubble thermal growth with a slip velocity between the bubble and the liquid phase: ... [64] Eq. 11 without slip, -- [65] Eq. 12, direct numerical simulations of [57] for $: J a=1: \circ R e_{0}=0.1, \square R e_{0}=1, \triangle R e_{0}=10, \nabla R e_{0}=100 ; J a=10: \bullet R e_{0}=0.1, \square R e_{0}=1, \Delta R e_{0}=10$, $\boldsymbol{\nabla} R e_{0}=100$ and $-[68]$ Eq. 14 . 


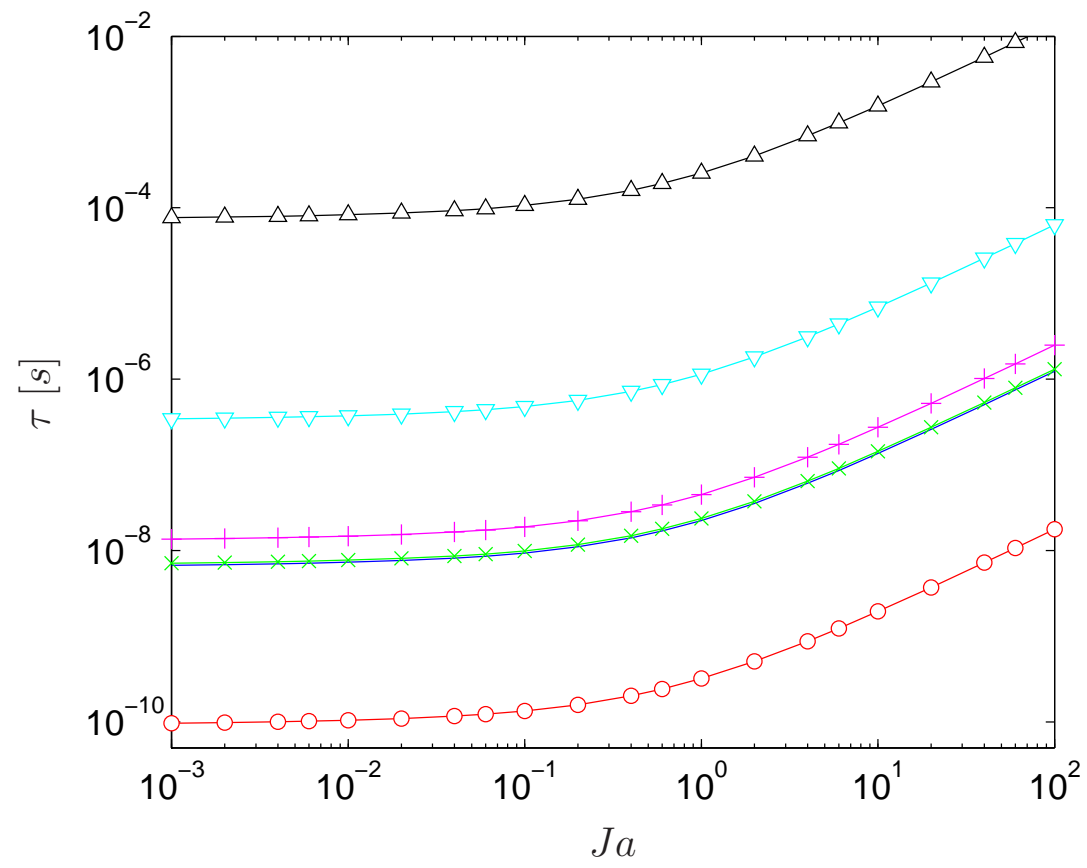

Figure 3: Time corresponding to the theoretical inception of thermal growth as a function of Jakob number for different fluids: $\mathrm{H}_{2} \mathrm{O}-\triangle$ at $T_{\text {ref }}=293 \mathrm{~K}$ and $-\nabla$ at $T_{\text {ref }}=355 \mathrm{~K},-\mathrm{R} 114$ at $T_{\text {ref }}=293 \mathrm{~K},-\times$ $N_{2}$ at $T_{\text {ref }}=78 \mathrm{~K},-\circ \mathrm{H}_{2}$ at $T_{\text {ref }}=23 \mathrm{~K},-+\mathrm{O}_{2}$ at $T_{\text {ref }}=90 \mathrm{~K}$. 
vapour production area

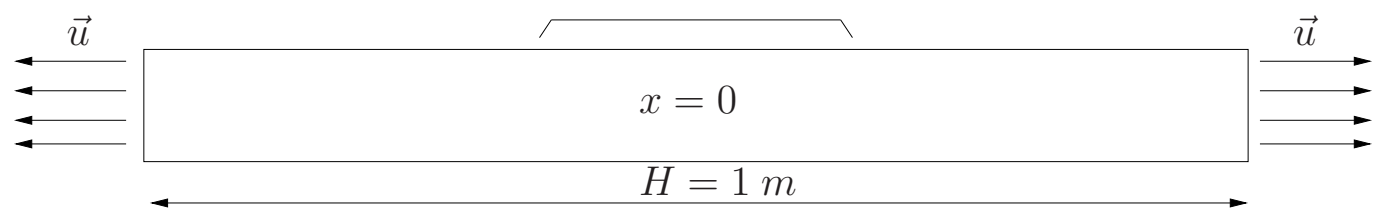

Figure 4: Expansion tube 1D test case. 


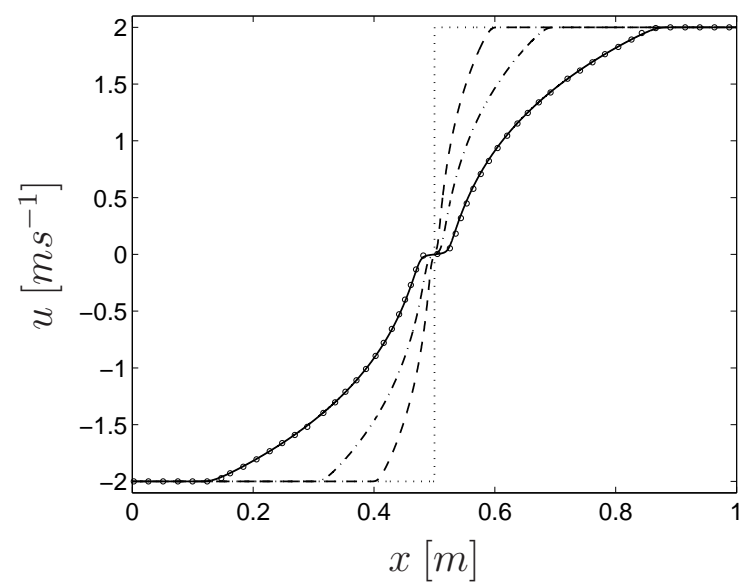

(a)

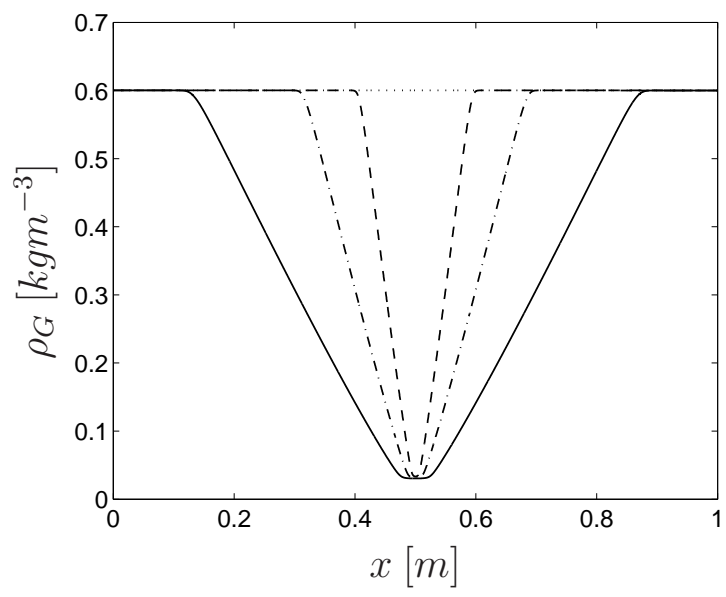

(c)

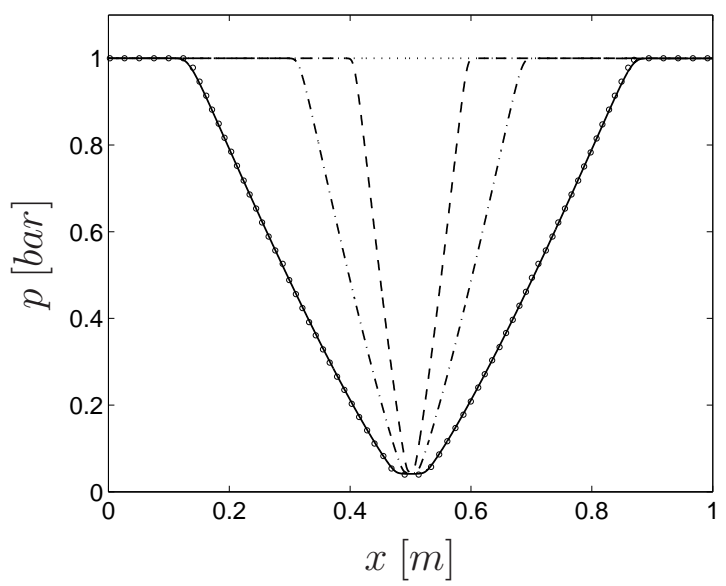

(b)

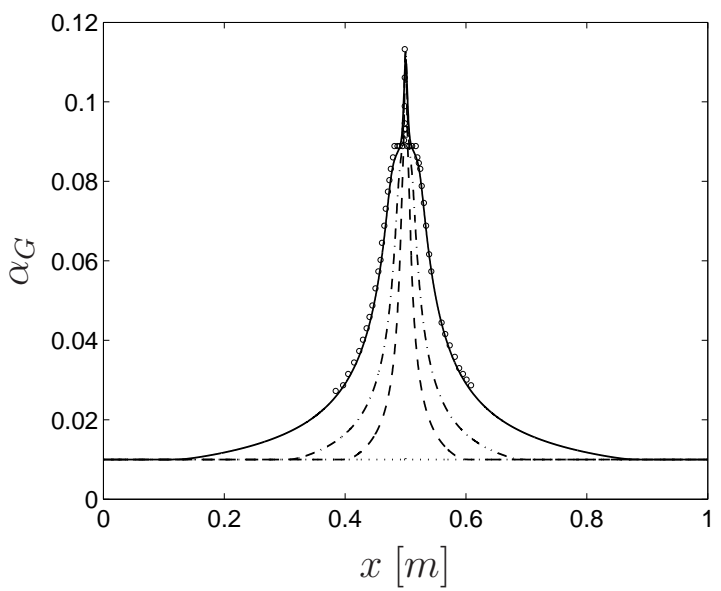

(d)

Figure 5: Results for water at $|u|=2 \mathrm{~ms}^{-1}$ without mass transfer $\left(\Gamma_{G}=0\right)$ : velocity (a), pressure (b), gas density (c) and gas volume fraction (d) versus position at time : ... $10^{-4} \mathrm{~ms},--0.8 \mathrm{~ms},-.-1.6 \mathrm{~ms}$ and $-3.2 \mathrm{~ms}$; $07 \mathrm{Eq}$. model [14] at $3.2 \mathrm{~ms}$. 


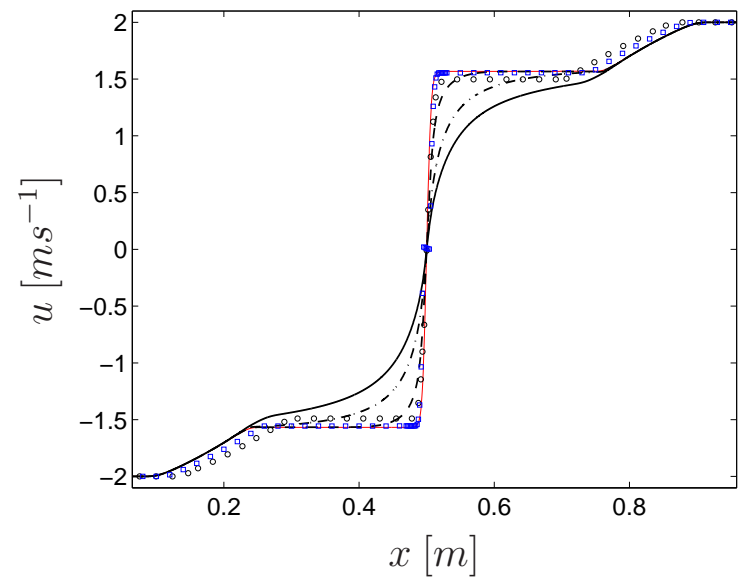

(a)

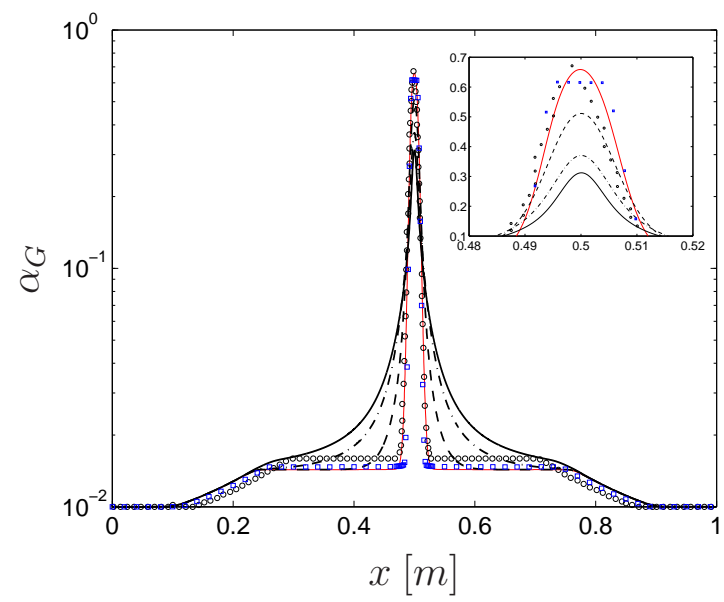

(c)

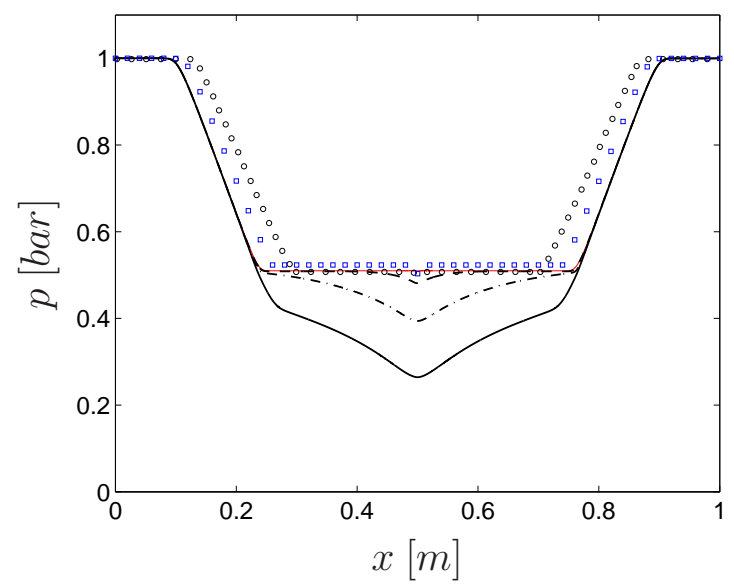

(b)

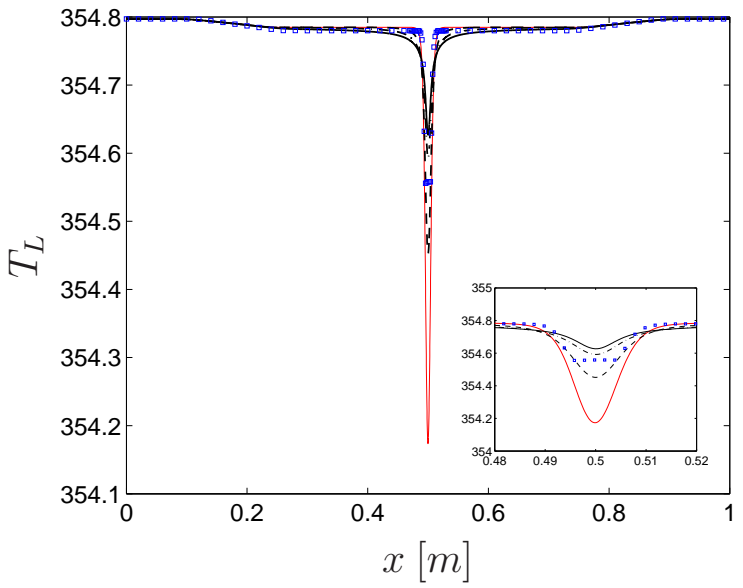

(d)

Figure 6: (colour online) Results for water at $|u|=2 \mathrm{~ms}^{-1}$ at time $3.2 \mathrm{~ms}$ : velocity (a), pressure (b), gas volume fraction (c), liquid phase temperature (d) versus position for : - inertial growth with $d_{b}=0.5 \mathrm{~mm}$ (Eq. 25); thermal growth (Eq. 24 and 14) with $--d_{b}=0.5 \mathrm{~mm},-.-d_{b}=3 \mathrm{~mm}$ and $-d_{b}=10 \mathrm{~mm}$; instantaneous phase change with $\circ 7$ Eq. model of [14] and $\square$ from [3] 4Eq. model with $d p^{\text {sat }} / d T=2000 \mathrm{PaK}^{-1}$ (Eq. 26). 


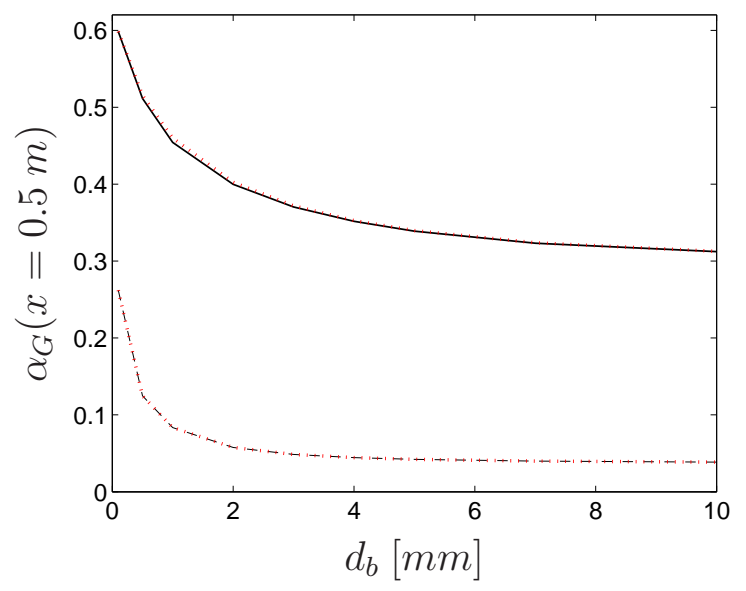

(a)

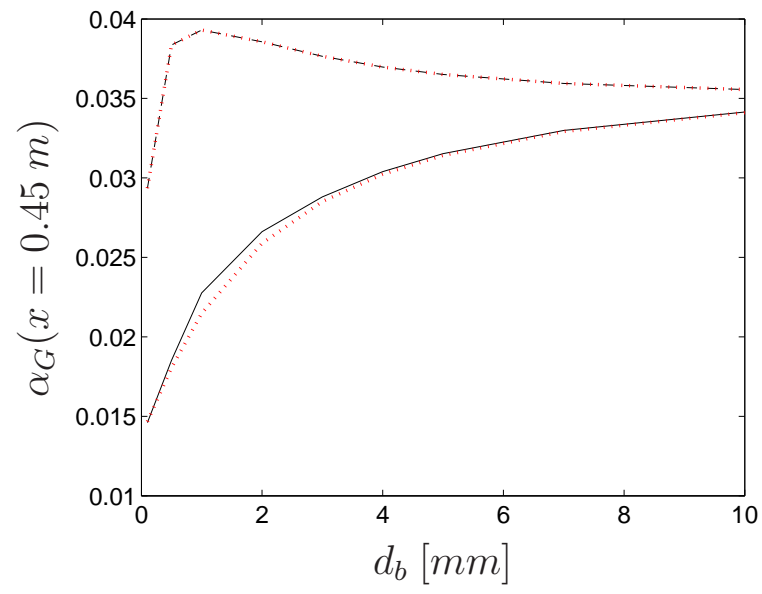

(b)

Figure 7: Gas volume fraction for $x=0.5 \mathrm{~m}$ and $x=0.45 \mathrm{~m}$ at $t=3.2 \mathrm{~ms}$ versus reference bubble diameter for test case with water and -- test case with R114; ... results obtained considering $N u_{L}=N u_{L 0}$ (Eq. 11). 


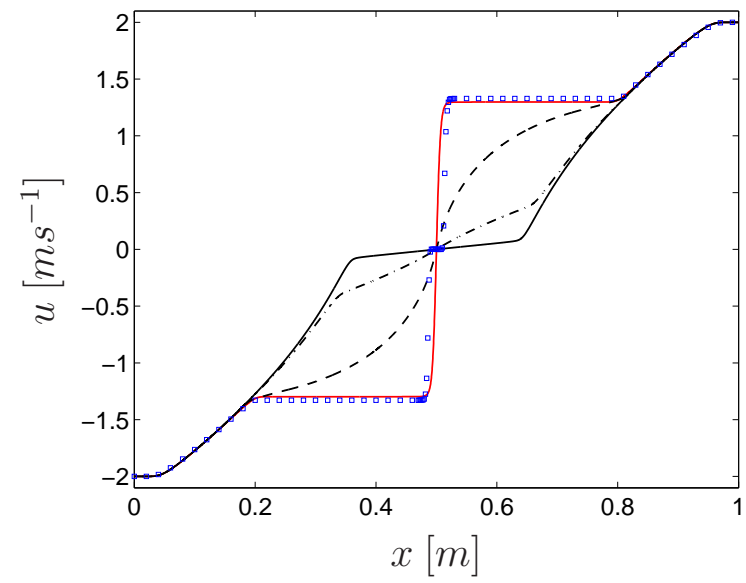

(a)

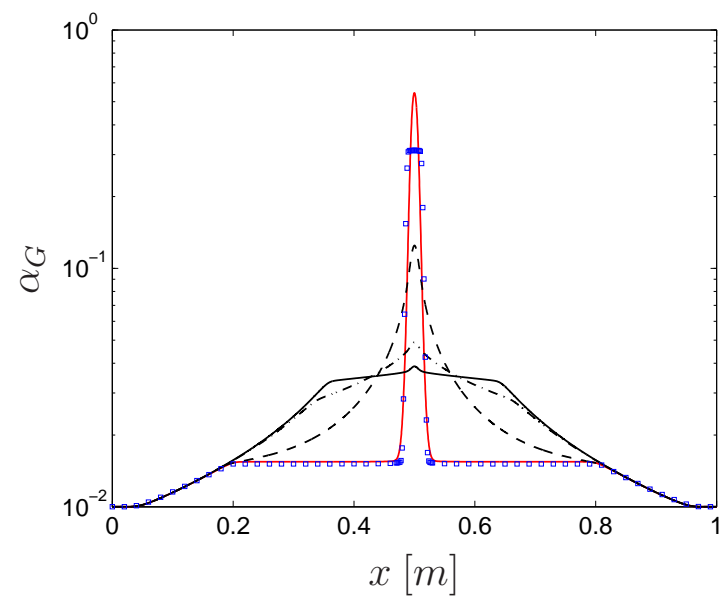

(c)

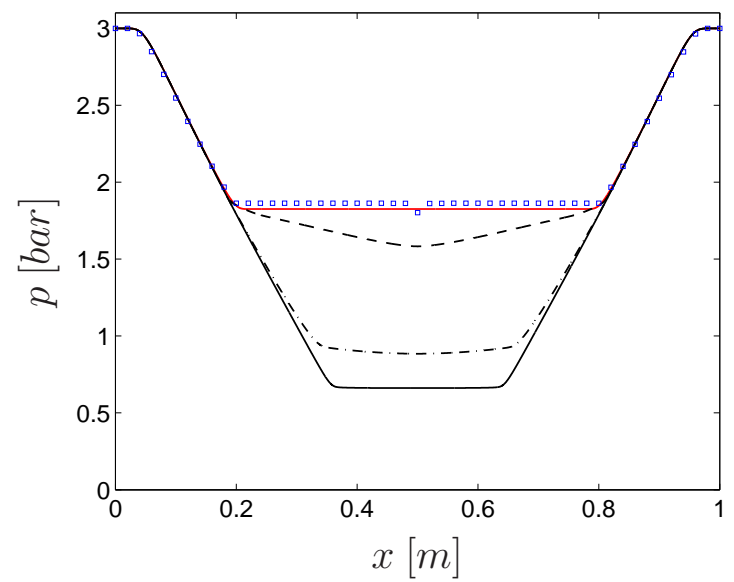

(b)

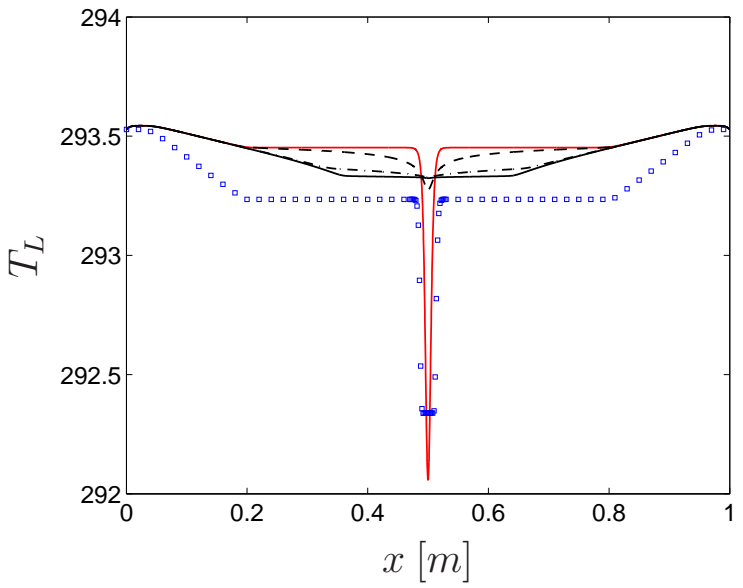

(d)

Figure 8: (colour online) Results for R114 at $|u|=2 \mathrm{~ms}^{-1}$ at time $3.2 \mathrm{~ms}$ : velocity (a), pressure (b), gas volume fraction (c), liquid phase temperature (d) versus position for : - inertial growth with $d_{b}=0.5 \mathrm{~mm}$ (Eq. 25); thermal growth (Eq. 24 and 14) with $--d_{b}=0.5 \mathrm{~mm},-.-d_{b}=3 \mathrm{~mm}$ and $-d_{b}=10 \mathrm{~mm}$; $\square$ instantaneous phase change from [16] 4Eq. model with $d p^{s a t} / d T=4720 \mathrm{PaK}^{-1}$ (Eq. 26). 


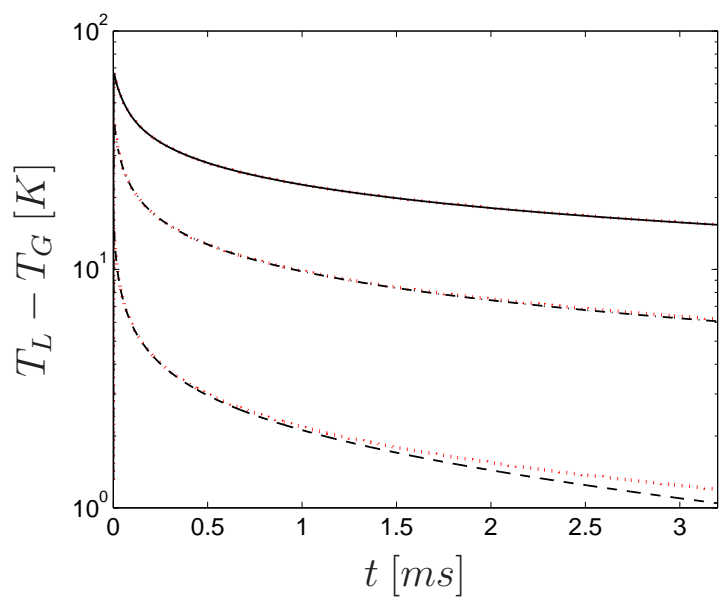

(a)

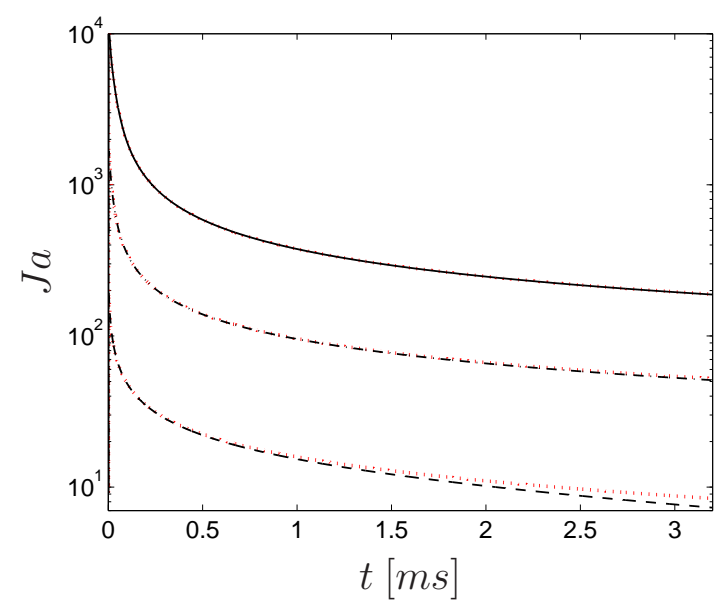

(c)

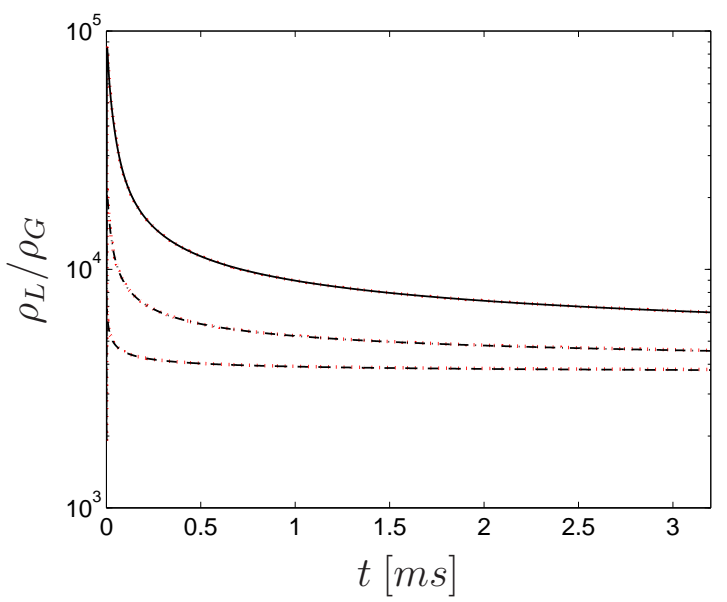

(b)

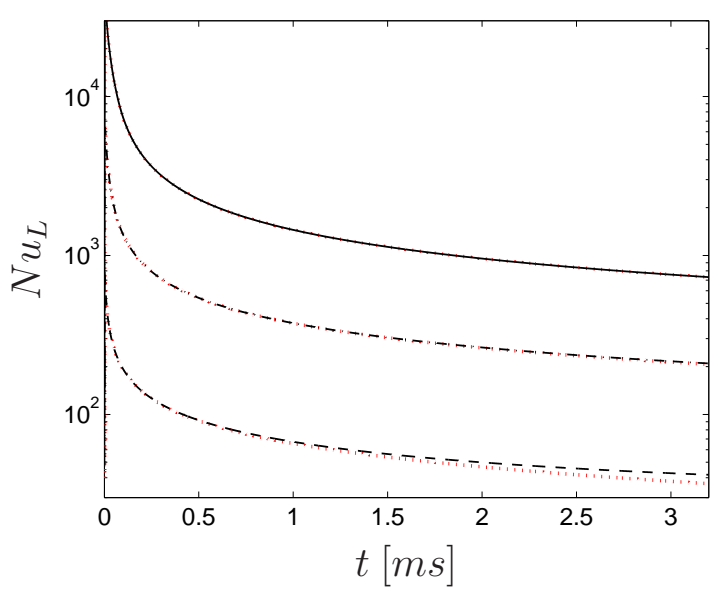

(d)

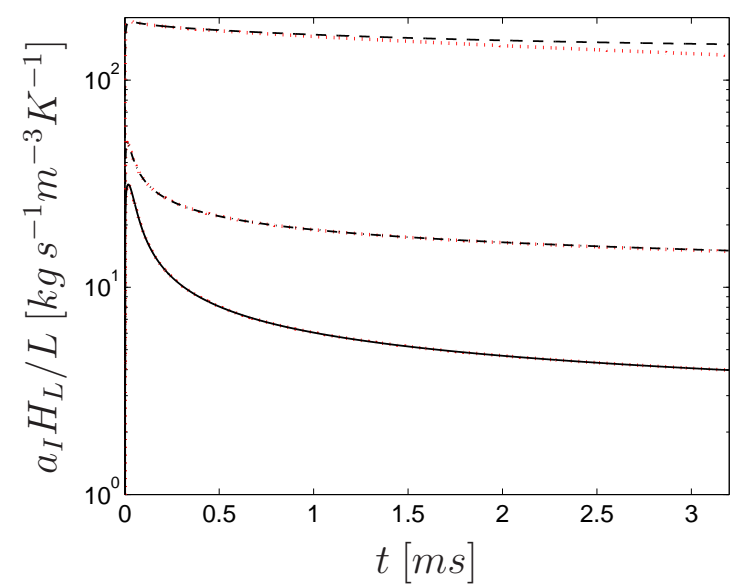

(e)

Figure 9: Influence of bubble diameter with water at $x=0.5 \mathrm{~m}$ for $|u|=2 \mathrm{~ms}^{-1}$ : (a) temperature difference, (b) density ratio, (c) Jakob number, (d) Nusselt number and (e) $a_{I} H_{L} / L$ versus time for $--d_{b}=0.5 \mathrm{~mm}$, -.$d_{b}=3 \mathrm{~mm},-d_{b}=10 \mathrm{~mm} ; \ldots$ results obtained considering $N u_{L}=N u_{L 0}$ (Eq. 11). 


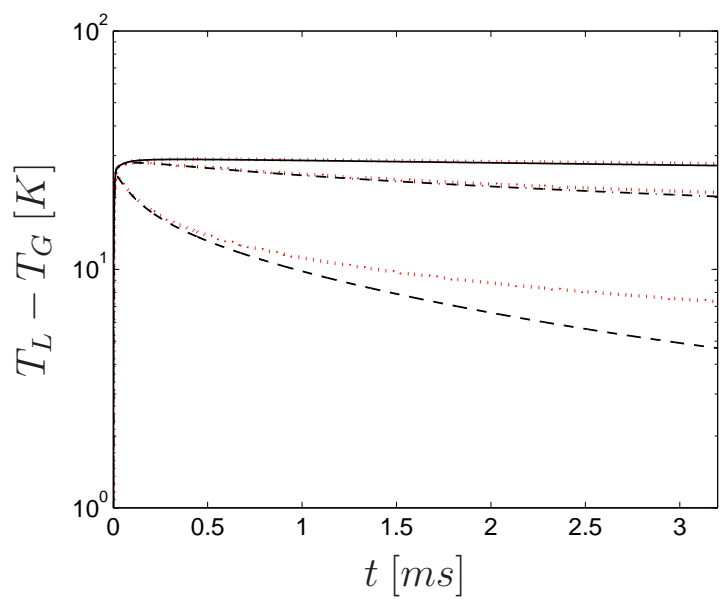

(a)

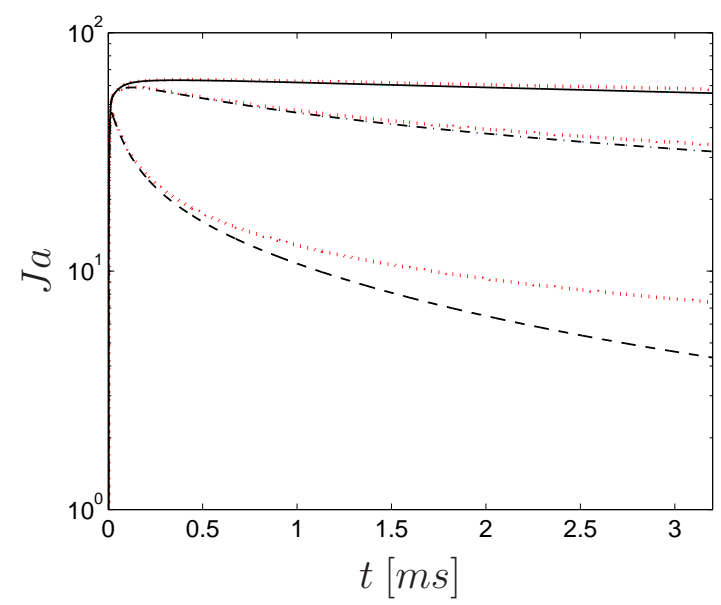

(c)

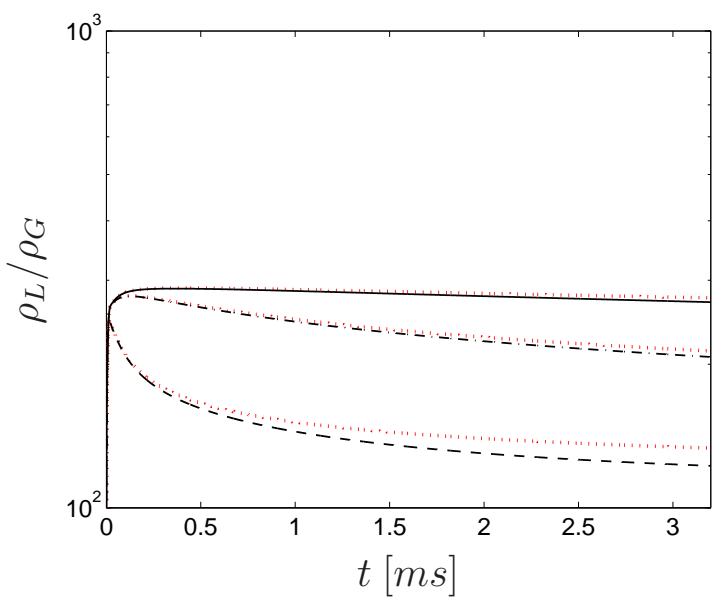

(b)

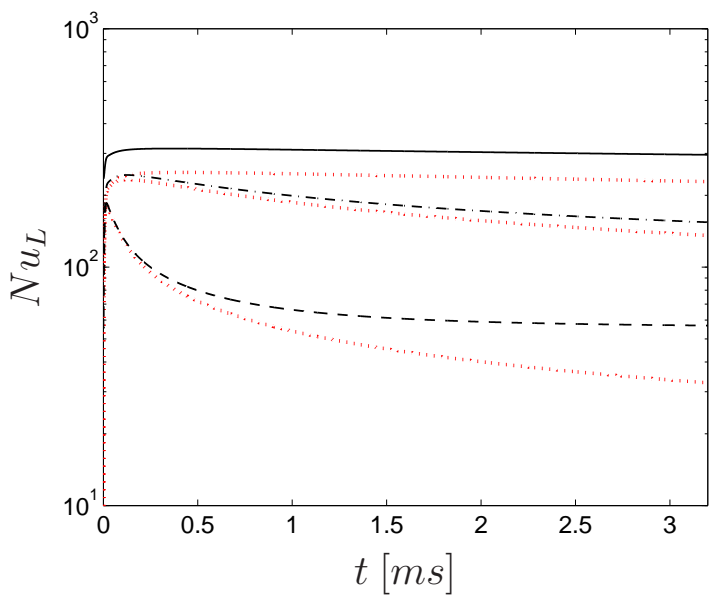

(d)

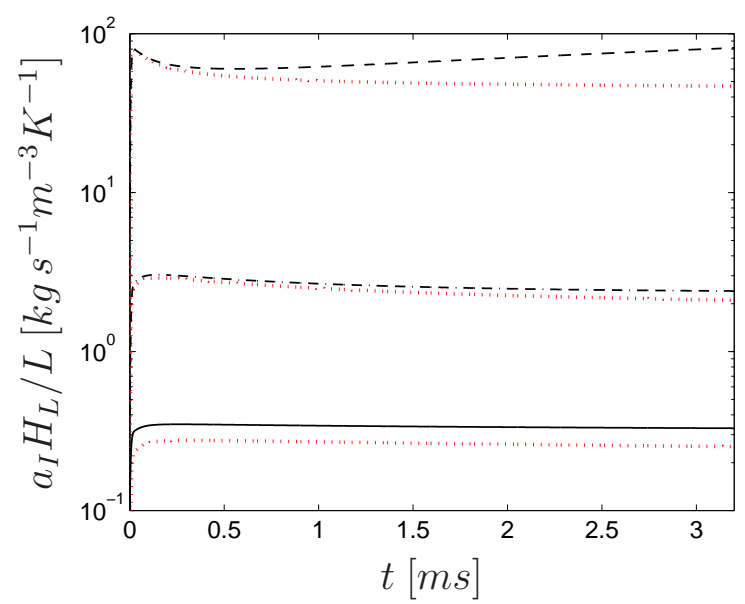

(e)

Figure 10: Influence of bubble diameter with R114 at $x=0.5 \mathrm{~m}$ for $|u|=2 \mathrm{~ms}^{-1}$ : (a) temperature difference, (b) density ratio, (c) Jakob number, (d) Nusselt number and (e) $a_{I} H_{L} / L$ versus time for $--d_{b}=0.5 \mathrm{~mm}$, -.$d_{b}=3 \mathrm{~mm},-d_{b}=10 \mathrm{~mm} ; \ldots$ results obtained considering $N u_{L}=N u_{L 0}$ (Eq. 11). 


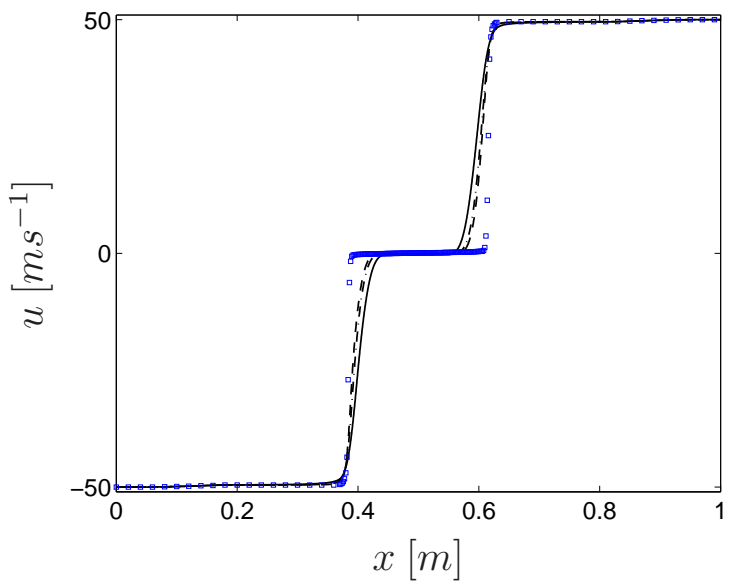

(a)

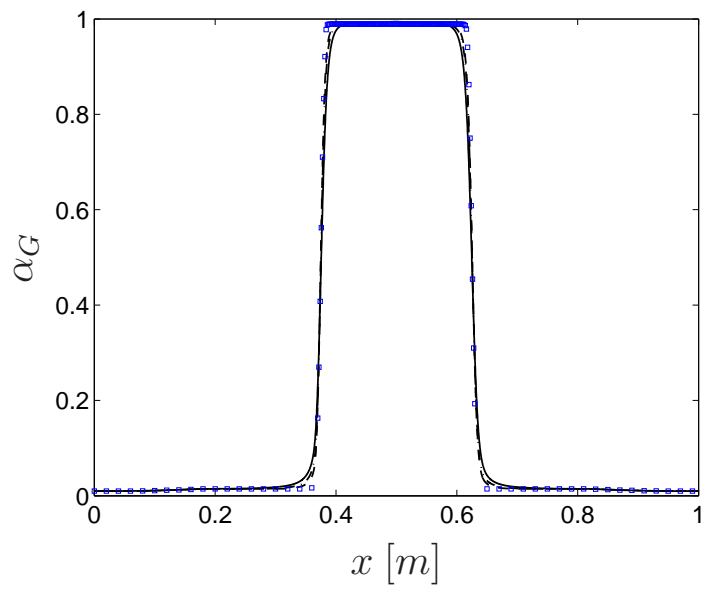

(c)

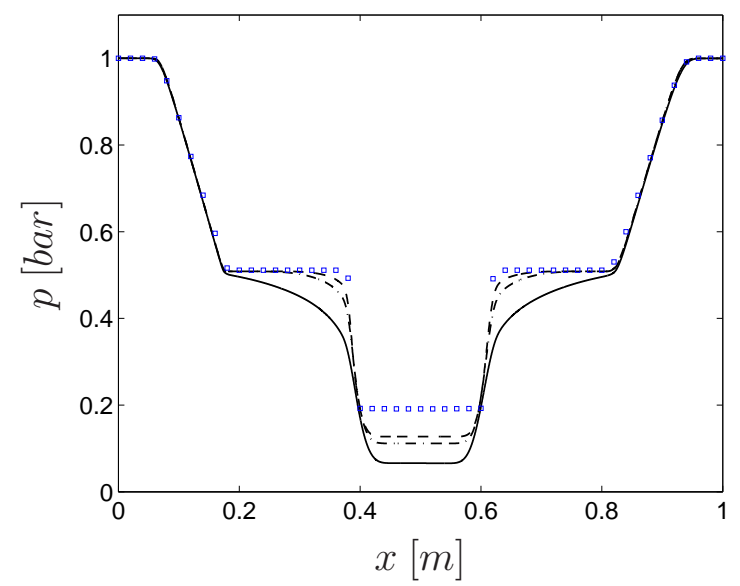

(b)

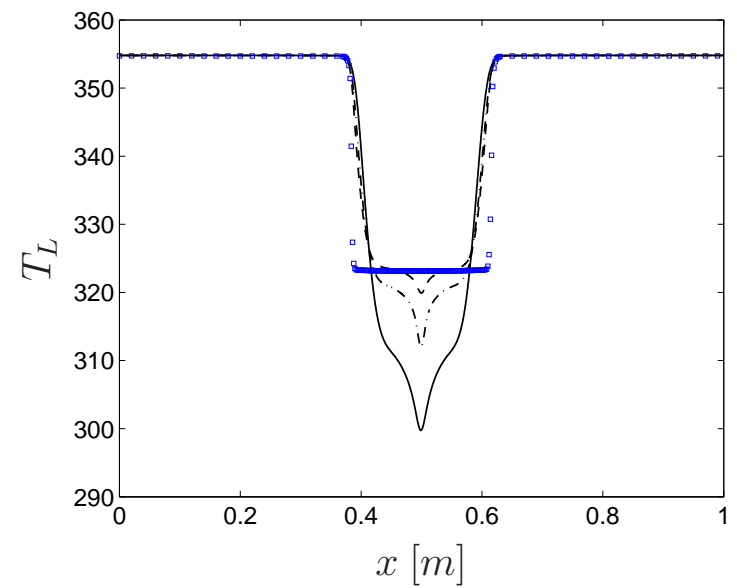

(d)

Figure 11: Results for water at $|u|=50 \mathrm{~ms}^{-1}$ at time $2.5 \mathrm{~ms}$ : velocity (a), pressure (b), gas volume fraction (c), liquid phase temperature (d) versus position for : thermal growth (Eq. 24 and 14) with $--d_{b}=0.5 \mathrm{~mm},-.-d_{b}=1 \mathrm{~mm}$ and $-d_{b}=3 \mathrm{~mm}$; instantaneous phase change with $\square$ Eq. 26 with $d p^{\text {sat }} / d T=1000 \mathrm{KPa}^{-1}$. 